\title{
Kalium-Argon-Daten zum Alter des Laacher Vulkanismus, der Rheinterrassen und der Eiszeiten
}

\author{
Von J. Frechen, Bonn und H. J. Lippolt, Heidelberg \\ Mit 8 Abbildungen im Text
}

$\mathrm{Z}$ us a mmenfassung. Die Vulkanite des Laacher-See-Gebietes in der Eifel können mit den Terrassenbildungen des Rheines zeitlich korreliert und ihre Föderfolge auf diese Weise stratigraphisch festgelegt werden. Dadurch bietet sich eine Möglichkeit, die K-Ar-Datierungsmethode an Proben von pleistozänem Alter zu testen und zu prüfen, ob die physikalisch bestimmte Sequenz mit der geologischen übereinstimmt. Datierungen wurden vorgenommen an 13 Sanidinen, 7 Biotiten, 2 Augiten und 14 Gesteinen der Eifel, ferner als Ergänzung an 6 Gesteinen der Umgebung von Agde, Dept. Hérault, Südfrankreich. Die meisten Datierungen ergaben geologisch wahrscheinliche Alter. Sie reichen in der Eifel von $570.103 \mathrm{a}$ bis $100.103_{\mathrm{a}}$, bei Agde von $1400.103 \mathrm{a}$ bis $640.10^{3} \mathrm{a}$. Diffusionsexperimente an Sanidinen und Biotiten zeigten, daß das atmosphärische Argon durch Ausheizen nicht zufriedenstellend entfernt werden kann, ohne daß radiogenes Argon verloren geht. Eine Kontrollprobe des abgeheizten radiogenen Gases mit radioaktivem $\mathrm{Ar}^{39}$ wurde getestet und erwies sich als nützliche Korrekturgröße.

$\mathrm{Sum} \mathrm{m}$ a r y : The volcanic rocks of the Laacher-See-area in the Eifel can be correlated chronologically with the formation of terraces by the River Rhine. Thus the sequence of these rocks becomes stratigrafically determinable. This offers a possibility for testing the $\mathrm{K}$-Ar-dating-method with samples of Pleistocene age and for checking the correspondance of the physically determined sequence and the geological sequence. Specimens of 13 sanidines, 7 biotites, 2 augites, 14 rocks of the Eifel, and - in addition -6 rocks of the environment of Agde, Dept. Hérault, in southern France were dated with geological likely ages resulting in most cases. In the Eifel they reach from 570.103a to $100.103 \mathrm{a}$, at Agde from 1400.103a to 640.103a. Experiments of diffusion with sanidines and biotites demonstrated, that atmospheric Argon cannot be sufficiently removed by heating without loss of radiogenic Argon. A control-sample of the heated radiogene gas with radioactive $\mathrm{Ar}^{39}$ was tested and proved to be a usful correction-factor.

\section{Einleitung}

Die K-Ar-Methode ist derzeit die einzige an älterem Material erprobte Datierungsart, mit der pleistozäne Alter bis hin zum Anwendungsbereich der C14-Methode bestimmt werden können. $\mathrm{Die} \mathrm{U} / \mathrm{Th}$-, $\mathrm{Pb}$ - und die Rb-Sr-Methoden lassen sich bei Proben, die jünger sind als das obere Tertiär, nicht mehr verwenden.

Schon 1956 haben Curtis \& al. zwei Biotite aus dem obersten Pliozän datiert und ein Alter von 1,7 Ma gefunden. Wesentlich genauer, da mit weniger Luftargonbeimischung versehen, wurde eine Sanidinmessung von Evernden \& al. (1957) mit 0,96 Ma, die wahrscheinlich eine frühe pleistozäne Vereisung datiert. In derselben Arbeit wurde anhand von zwei Datierungen an jungvulkanischen Sanidinproben aus der Eifel gezeigt, daß K-ArMessungen unter $1 \mathrm{Ma}$ grundsätzlich möglich sind.

Mit Hilfe von K-Ar-Datierungen haben Gentner \& Z Z̈̈hringer (1960, 1963) die Tektite in drei Gruppen eingeteilt. Die jüngste Gruppe mit einem mittleren Alter von $0.72 \pm 0.06 \mathrm{Ma}$ könnte als Leitfunde für die oberen Trinilschichten im indo-australischen Raum dienen. V. Koenigswald \& al. (1961) haben für einen post- oder spättrinilen Tephrit von Java $0.05 \pm 0.06 \mathrm{Ma}$ angegeben, was mit dem Tektitenergebnis in Einklang ist.

Weitere K-Ar-Alter von Leakey \& al. (1961) und von v. Koenigswald \& al. (1961) beziehen sich auf das Alter des Olduvai-Menschen. Nach den Ergebnissen von LEAKEy \& al. müßte die Grenze Pliozän/Pleistozän bei ca. 2 Ma liegen. Es scheint aber bei diesem Alterswert Vorsicht geboten, weil die Bestimmung an Mineralen aus Tuffen vorgenommen wurde. Wie gezeigt wird, haben sich bei solchen Mineralen wiederholt überhöhte K-ArAlter ergeben. 
J. F. Evernden, G. H. Curtis und Mitarbeiter nehmen mit ca. 3.106a sogar ein noch höheres Alter für den Beginn des Villafranca an. Sie stützen sich u. a. auf K-Ar-Datierungen von G. B. Dalrymple (1963) an jungtertiären Gesteinen in der Sierra Nevada. Einen weiteren Beitrag zu dieser Frage lieferten in Verbindung mit den K-Ar-Daten Messungen des Paläomagnetismus der untersuchten Gesteine (A. Cox \& al.). Sie zeigten bis zu $1.10^{6} \mathrm{a}$ eine entgegengesetzte und von 2 bis über $3.10^{6} \mathrm{a}$ wieder die normale Richtung des Magnetismus.

Im Laacher-See-Gebiet in der Eifel haben im Pleistozän über eine Zeit von mehr als einer halben Million Jahre vulkanische Ausbrüche stattgefunden, die vor ungefähr 10000 Jahren zum Abschluß kamen. Da eine Verknüpfung dieses Vulkanismus mit dem Terrassensystem des Rheines möglich ist und so ein Anschluß an die Pleistozän-Stratigraphie gefunden werden kann, lag es nahe, an seinen Förderprodukten Datierungen durchzuführen.

Altere pleistozäne Vulkane sind aus der Umgebung von Agde, Dept. Hérault, Südfrankreich, bekannt. Hier können einige Vorkommen stratigraphisch dem Villafranca zugeordnet werden. Ihre Datierung ist wichtig für die Frage, wo die zeitliche Grenze zwischen dem Pliozän und dem Pleistozän liegt.

Das Ziel dieser Arbeit ist es, festzustellen, welche Faktoren die K-Ar-Methode nach kleinen Altern hin begrenzen, wieweit die geologisch festgelegte Abfolge des Laacher Vulkanismus sich in den Altern der vulkanischen Minerale und Gesteine abzeichnet und welche Aussagen sich für die Entstehungszeit der Rheinterrassen und damit unter $\mathrm{Zu}$ grundelegung geeigneter Annahmen für den Ablauf der Eiszeiten ergeben.

\section{Das Meßverfahren}

Der Kaliumgehalt der untersuchten Proben wurde flammenphotometrisch bestimmt. In die Tabellen sind die reinen $\mathrm{K}$-Gehalte eingetragen und nicht die in geochemischen Arbeiten oft gebrauchten $\mathrm{K}_{2} \mathrm{O}$-Werte. Alle $\mathrm{K}$-Angaben sind Mittelwerte aus mindestens zwei Bestimmungen.

Die Argongehalte sind massenspektrometrisch bestimmt worden. Nach Homogenisierung des Probenmaterials durch Zerkleinern und Aussieben einer geeigneten Korngröße (je nach der Einwaage) wurde dieses in einem Molybdäntiegel induktiv geschmolzen. Bei der verwendeten Hochfrequenz mit niederohmigem Ausgang (400 K Hz) setzte keine Glimmentladung im Schmelzofen ein. Die erreichte Höchsttemperatur liegt bei $2000^{\circ} \mathrm{C}$. Nur bei der Datierung der Sanidinbomben wurden große Stücke geschmolzen, wodurch der atmosphärische Argonanteil besonders klein blieb. Sowohl die Argon-Extraktionsapparatur als auch das Massenspektrometer sind ausheizbar. Die Proben befinden sich vom Beginn der Meßreihe an innerhalb des Ausheizofens und werden zusammen mit der Apparatur während $15 \mathrm{~h}$ bei Temperaturen von $150^{\circ}$ bis $200^{\circ}$ ausgeheizt. Je nach der Art der Proben wurde dieses Ausheizen anschließend bei höherer Temperatur fortgesetzt, $300^{\circ} \mathrm{da}-$ bei jedoch nur bei den Biotiten überschritten. Die Vorreinigung der extrahierten Gase erfolgt mit glühenden Zirkonspänen. Während des Schmelzens wird das freigegebene Argon an gekühlter Aktivkohle adsorbiert, um der Gefahr von Argonverlusten durch den Schmelzvorgang (Spiegelbildungen) zu begegnen. Danach wird das vorgereinigte Argon mittels Aktivkohle in den Feinreinigungsteil gepumpt und hier über heißem $\mathrm{Ca}$ und $\mathrm{Cu}-\mathrm{CuO}$ nachgereinigt, dann ein Kühlfinger auf die Temperatur des festen $\mathrm{CO}_{2}$ gebracht, der $\mathrm{Ca}$-Ofen abgeschaltet und abgekühlt und das Ar über einen Metallhahn in das Spektrometer eingelassen, wo zuerst die isotopische Häufigkeit bestimmt und anschließend die Argon-40-Menge mit einer kalibrierten atmosphärischen Argon-Menge verglichen wird. Jeder Messung geht ein Leeraufschluß voraus, um den Zustand der Apparatur zu überprüfen. Nach frischem Füllen der beiden Feinreinigungsöfen muß man mit $5.10^{-8} \mathrm{~cm}^{3}$ atmosphärischem Argon je Versuch rechnen, wenn die beiden Ơfen je eine 
Stunde heiß sind und der Schmelztiegel 1/4h maximal geheizt wurde. Nach einer Woche Ausheizen der Ófen betrug der Leeraufschluß unter obigen Bedingungen $1.10^{-8} \mathrm{~cm}^{3}$, wobei 0.6 auf den $\mathrm{Ca}-\mathrm{Ofen}, 0.1$ auf den $\mathrm{Cu}-\mathrm{Cu}-\mathrm{O}-\mathrm{O}$ en und 0.3 auf den Schmelztiegel entfielen.

Durch kürzere Reinigungszeiten ließ sich dieser Betrag in einigen Fällen weiter verringern.

\section{Diffusions- und Entgasungsversuche}

Als wir darangingen, uns mit der Chronologie des Eifelvulkanismus und des rheinischen Terrassensystems zu beschäftigen, war noch recht wenig über die Argonverluste in Sanidinen, Biotiten und Gesteinsproben bekannt. Schon die U-Th/He-Methode hatte große Schwierigkeiten bereitet, weil die verschiedenen Minerale ein unterschiedliches Speichervermögen für $\mathrm{He}$ haben (KEEviL 1942). GerLING (1939) hat deswegen vorgeschlagen, die Aktivierungsenergie des $\mathrm{He}$ im Mineral als Kriterium für die Brauchbarkeit desselben zur Altersbestimmung zu verwenden. Bei K-Ar-Datierungen hatten WetherILL \& al. (1955) große Diskrepanzen zwischen den Glimmer- und Feldspataltern festgestellt. GENTNER \& KLEY (1957) fanden dagegen an ihren Schwarzwaldproben nur unbeträchtliche Unterschiede, sie stießen aber auf den schon von Gray (1909) bei Helium-Datierungen bemerkten Effekt, daß die Proben beim Zerkleinern Edelgas verlieren können. Als Ursache wurde von NoDDACK \& ZEITLER (1956) aufgrund eines Entgasungsexperimentes an einer Orthoklasprobe zwischen $500^{\circ}$ und $1000^{\circ} \mathrm{C}$ die Anreicherung des radiogenen Argons an den neuen Grenzflächen angesehen. GerLing \& Morozova (1957) konnten jedoch ihre Experimente an Glimmern mit Volumendiffussion des Argons erklären. Die gefundenen Aktivierungsenergien von 57 bis $85 \mathrm{kcal} / \mathrm{Mol}$ kennzeichneten die untersuchten Minerale als zur Altersbestimmung brauchbar. In ähnlicher Weise zeigte REynolDs (1957), daß sich die Feldspat-Glimmer Diskrepanz durch schwächere Speicherung des Argons im Feldspatkristall deuten läßt.

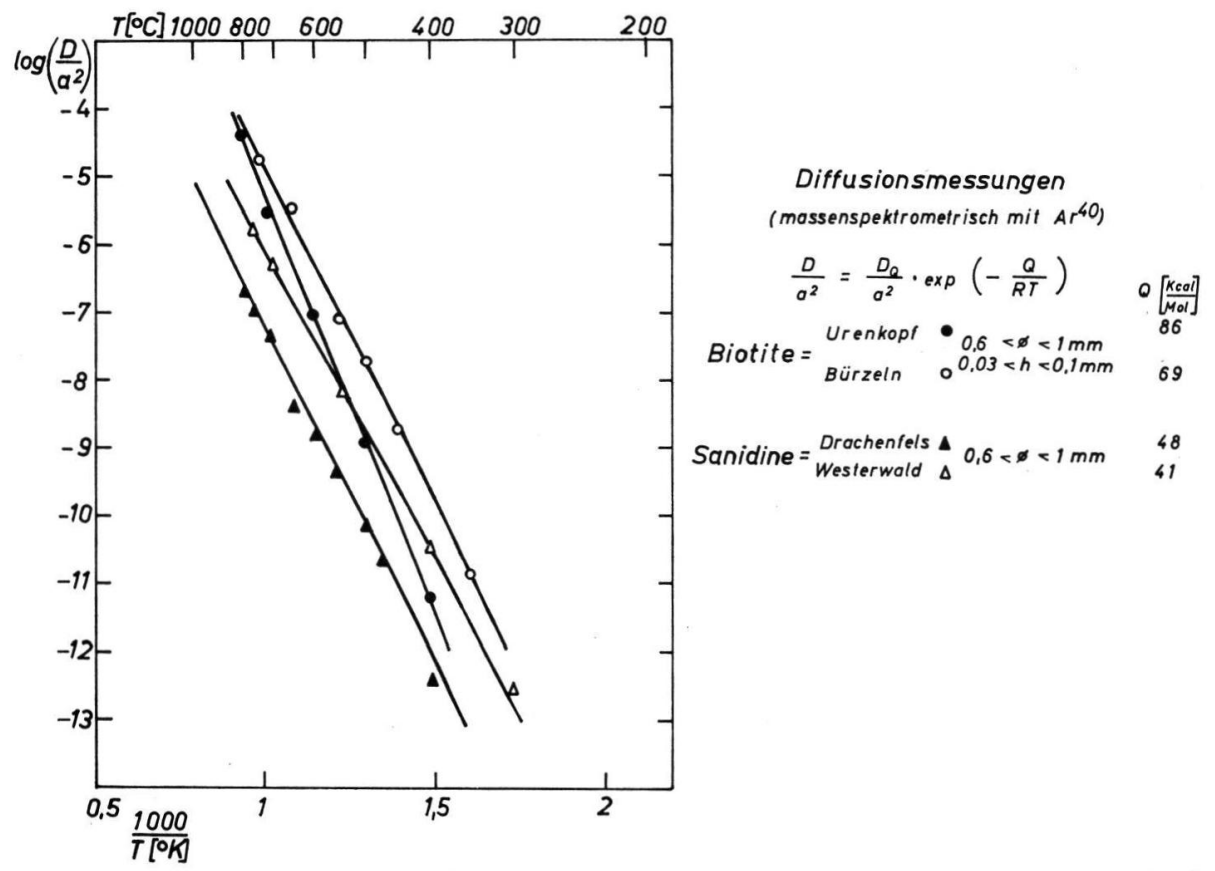

Abb. 1. D/a 2 -Werte für zwei Sanidine und zwei Biotite in Abhängigkeit von der reziproken Temperatur. 
Um sicher zu sein, daß die zur Messung vorgesehenen Sanidine nicht dieselben Verluste wie die anderen Feldspate erleiden, wurde an zwei Biotiten, zwei Sanidinen und einer Gesteinsprobe untersucht, wie die $\mathrm{D} / \mathrm{a}^{2}$-Kurven für Sanidine im Vergleich mit denen von Biotiten verlaufen, ferner, ob die Argonisotope $\mathrm{Ar}^{40}$ und $\mathrm{Ar}^{36}$ auf dieselbe Weise vom Kristall festgehalten werden, d. h. das atmosphärische Argon gleichmäßig über den Kristall verteilt ist, ob es in einer Randschicht sitzt oder gar nur adsorbiert ist. Der eine Biotit stammte vom Tuffschlot Bürzeln in der Schwäbischen Alb (voN Engelhardt \& WeISKIRCHNER 1961), der andere vom Steinbruch Urenkopf bei Haßlach im Schwarzwald. Die Sanidine sind tertiären Alters (Frechen \& Lippolt, 1965) und stammen aus dem Siebengebirge und Westerwald (Drachenfels und Kärlich).

Die Proben wurden durch Sieben auf eine einheitliche Korngröße gebracht und in einem Quarzbecher in den heißen Teil eines Molybdänofens getaucht, sobald dieser die gewünschte Temperatur hatte. Diese wurde mit einem Thermoelement gemessen und mittels eines Fallbügelreglers konstant gehalten. Die innerhalb einer vorgegebenen Zeit freigewordenen Argonmengen wurden gereinigt und massenspektrometrisch gemessen.

Die Berechnung der D/a ${ }^{2}$-Werte erfolgte wie bei FECHTIG \& al. (1961) nach den von WRAGE (1962) angegebenen Formeln zur Berücksichtigung der fortschreitenden Randverarmung der Kristalle. Dabei wurden die hierzu notwendigen Voraussetzungen streng eingehalten. Die Ergebnisse sind in Abbildung 1 festgehalten.

Die Kurven wurden bis $20^{\circ}$ extrapoliert und für diese Temperatur die Alterskorrekturparameter (Fechtig \& al. 1960, Wrage 1962) bestimmt. Die ermittelten Werte enthält die Tabelle 1.

Tabelle 1

Alterskorrekturparameter $\mathrm{d}$ bei Zimmertemperatur $(\mathrm{S}=$ Sanidin, $\mathrm{B}=$ Biotit $)$

\begin{tabular}{lcccc}
\hline Probe & $\begin{array}{c}\text { Alter } \\
106 \mathrm{a}\end{array}$ & $\begin{array}{c}\text { Akt.Energie } \\
\mathrm{kcal} / \mathrm{Mol}\end{array}$ & $\begin{array}{c}\mathrm{D} / \mathrm{a}^{2}\left(20^{\circ} \mathrm{C}\right) \\
\mathrm{sec}^{-1}\end{array}$ & $=\mathrm{d} \frac{\sqrt{\lambda . \mathrm{a}^{2}}}{\zeta}$ \\
\hline Kärlich & $\mathrm{S} 23$ & 41 & $4.10-28$ & 2.105 \\
Drachenfels & $\mathrm{S} 23$ & 48 & $3.10-33$ & 7.107 \\
Bürzeln & B 20 & 69 & $10-29$ & 106 \\
Urenkopf & B 355 & 86 & $10-34$ & 108
\end{tabular}

Aus ihnen folgt, daß im Vergleich mit den Glimmern die Ergebnisse bei den Sanidinen schlechter sind, d. h. bei ihnen eher mit Verlusten durch Volumendiffusion zu rechnen ist. Eine eindeutige Antwort gibt darauf die Arbeit von BaAdsgand, Lipson \& Folinsbee (1960), nach der sieben Sanidin-Glimmerpaare innerhalb der Fehlergrenzen gleiche Alter ergeben haben.

Aus der Tabelle 1 kann entnommen werden, daß die vier Proben bei einer Lagertemperatur von $10-20^{\circ} \mathrm{C}$ kein radiogenes Argon durch Volumendiffusion verlieren. Erst Temperaturen von $150^{\circ}$ bis $200^{\circ} \mathrm{C}$ könnten während geologischer Zeiträume kritisch werden. Von verschiedenen Seiten wurde darauf hingewiesen, daß die wesentlichen Argonverluste durch Gefügeänderungen der Kristalle verursacht werden und nicht durch Volumendiffusion (Aмirkhnov \& al. 1959, Fechtig \& al. 1961), es sei denn, man untersucht Glaukonite oder Salze (Evernden \& al. 1960).

Die zweite Frage bezog sich auf die Bindung des atmosphärischen Argon. Sie wurde schon von Evernden \& al. (1960) angeschnitten. In der Abbildung 2 ist die diffundierte radiogene Argonmenge über der abgeheizten atmosphärischen Argonmenge aufgetragen.

Die Auswertung bereitet einige Schwierigkeiten, weil das atmosphärische Argon aus zwei Anteilen besteht. Der eine stammt von oder aus der Probe, der zweite ist das Argon des Leerversuches. Dieser zweite Anteil kann nur indirekt gemessen werden, indem man 
vorher und nachher einen Leerversuch ausführt und die mittlere Argonmenge von der Diffusionsmenge abzieht. Die Unsicherheit dieses Verfahrens bedingt einen erhöhten Fehler, der sich beim Addieren der Mengen aufsummiert.

Das atmosphärische Argon wird langsamer ausgetrieben, als wenn es nur adsorbiert wäre, aber schneller, als es bei gleichmäßiger Verteilung über den Kristall erwartet werden könnte. Im letzten Falle sollte es wie das radiogene Argon diffundieren. Dies führt zur folgenden Vorstellung: Nach der Bildung kommen die Minerale oder Gesteine in Berührung mit der Atmosphäre. $\mathrm{Da}$ sie meistens noch hohe Temperaturen haben, kann Argon wegen der erhöhten Diffusionskonstanten aus einer adsorbierten Schicht in die Oberfläche hineindiffundieren. Es bildet sich eine Verteilung aus, die sich nach der Abkühlung der Proben nicht mehr wesentlich ändert, weil die Diffusionskonstante zu klein ist.

Durch Ausheizen im Vakuum wird diese Verteilung geändert. Das diffundierte Gas wandert aber nicht nur zur Oberfläche, sondern auch in den Kristall hinein. Es ist deshalb nicht möglich, das atmosphärische Argon abzuheizen, ohne die radiogene Menge zu verfälschen. Der Abb. 2 ist zu entnehmen, daß mit 50\% Luft-Argon auch 10\% radiogenes Argon verloren gehen.

Die Kurven im rechten Teil der Abbildung 2 verdeutlichen die verschiedenen Verteilungen und die Richtung ihrer Veränderung zu den Zeiten $t_{0}$ bis $t_{3}$. Grundsätzlich müßten auch aus den diffundierenden Luftargonmengen Diffusionskonstanten berechnet werden können. Die mathematische Behandlung bereitet aber große Schwierigkeiten.

Zur Unterstützung der oben skizzierten Vorstellung, daß das Luftargon hineindiffundiert, wurde ein Temperaturexperiment durchgeführt. Dazu wurden in zwei Glasröhrchen je $0.1 \mathrm{~g}$ Sanidin (Drachenfels) eingeschmolzen (Argonfüllung der Röhrchen 607 torr bei $22^{\circ} \mathrm{C}$ ) und dann das eine $1.3 \mathrm{~h}$ auf $500^{\circ} \mathrm{C}$ und das andere $13 \mathrm{~h}$ auf $400^{\circ} \mathrm{C}$ erhitzt.

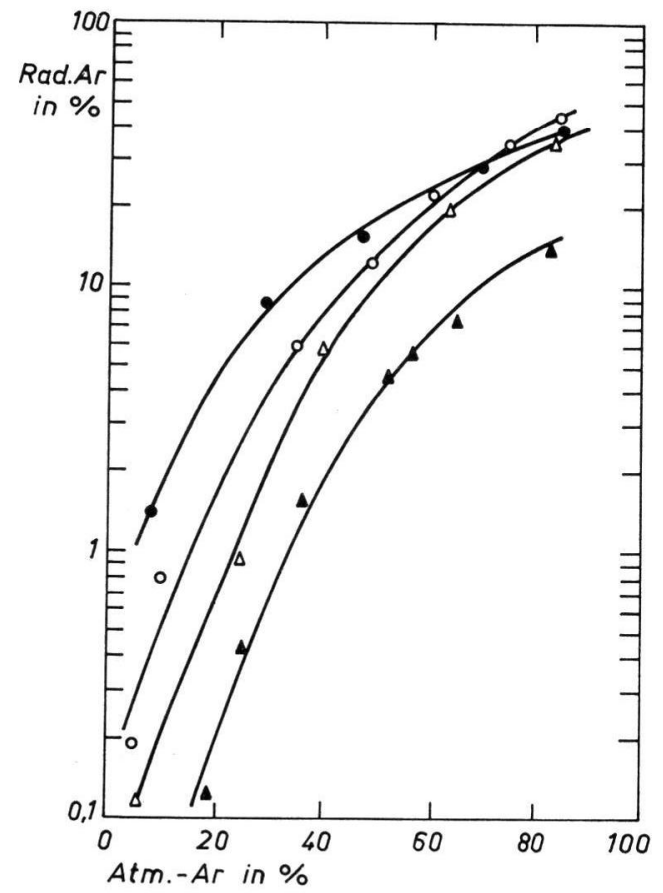

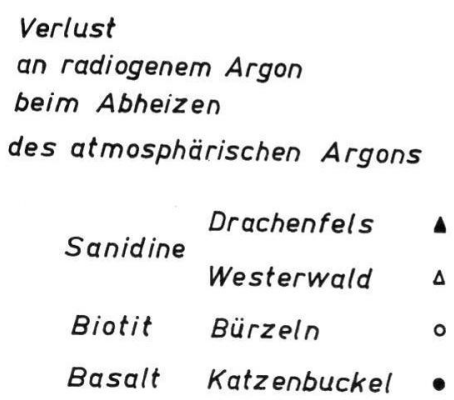

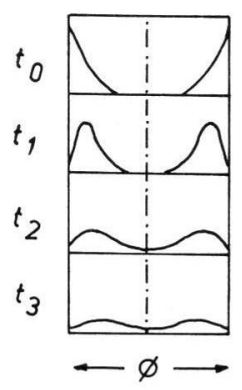

Abb. 2. Verlust an radiogenem Argon beim Abheizen des atmosphärischen Argons. Veränderung der Verteilung des Luftargons während des Abheizens. 
Die Korngröße der Sanidinkristalle lag zwischen 0.6 und $1 \mathrm{~mm}$. Es wurden 3.8 und 3.5.10-5 $\mathrm{cm}^{3}$ Argon/g Mat gemessen. Das ist $50 \mathrm{mal} \mathrm{mehr}$ als die unausgeheizte Probe Luftargon enthält. Macht man den Ansatz, daß die eindiffundierte Argonmenge proportional D.t sei, dann ergibt sich jedoch nur eine qualitative Übereinstimmung mit den gemessenen Diffusionskonstanten. Das Verhältnis der Mengen sollte 3.2 betragen, ist jedoch 1.1. Die Ursache dafür ist ungeklärt.

\section{Ausheizen mit Aktivierungskontrolle}

Nach den Ausführungen im vorigen Kapitel wird es nur möglich sein, die Proben durch Ausheizen hinreichend vom atmosphärischen Argon zu befreien, wenn auch die radiogenen Verluste unter Kontrolle gebracht werden. Das radiogene Argon wird sich sicher in der Nähe seiner Bildungsstätte befinden und von dort nach außen wandern. Dies kann mit $\mathrm{Ar}^{39}$ kontrolliert werden, das ebenfalls aus dem Kalium des zu datierenden Kristalls durch Neutronenaktivierung mittels der Reaktion

$$
\mathrm{K}^{39} \text { (n,p) } \mathrm{Ar}^{39} \quad \text { (260a, Halbwertzeit) }
$$

erzeugt wird. Man hat einmal die Möglichkeit, die während des Entgasens verlorene Ar ${ }^{39}$ Menge zu zählen, kann jedoch auch dieses Gas massenspektrometrisch erfassen. Unter der Annahme, daß sich die beiden Argonisotope $\mathrm{Ar}^{40}$ (rad) und $\mathrm{Ar}^{39}$ wegen der gleichen Muttersubstanz in ihrem Verhalten nicht unterscheiden, ist eine Abschätzung des experimentell verlorenen "Altersbetrages“ möglich. J. J. NAUGHTON hat vorgeschlagen, solches $\mathrm{Ar}^{39}$ aus zur Probe gemischten Kristallen zur Isotopenverdünnung zu verwenden.

Zur Überprüfung dieser Hypothese wurden im Münchener Reaktor drei Glimmer aus Eifelgesteinen bestrahlt und dann stufenweise im HF-Tiegel erhitzt. Die Tabelle 2 zeigt die Ergebnisse im Vergleich mit Messungen ohne Aktivierungskontrolle. Sie sind nicht genau, und es ist klar zu erkennen, daß bei diesen Glimmern der Gewinn in Anbetracht des Aufwandes sehr klein ist. Innerhalb der Meßgenauigkeit verhält sich nämlich das radiogene Argon wie das $\mathrm{Ar}^{39}$, aber leider auch das atmosphärische Argon, d. h. auch das

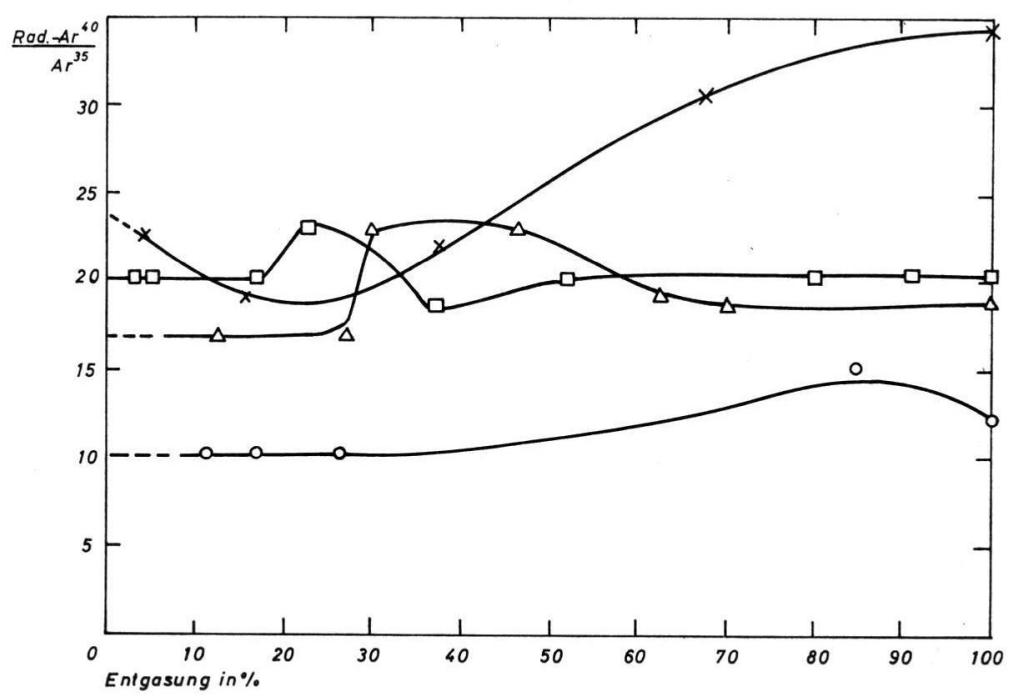

Abb. 3. Kontrolle der $A r^{40}(\mathrm{rad})-$ Verluste mit Ar39 Sanidin "Kärlich“ $A \mathrm{Ar}^{40}(\mathrm{rad})=6.8 .10^{-6} \mathrm{cc} / \mathrm{g}$ $0.28 \mathrm{~g}: 27 \%$ Aktiviert $0.21 \mathrm{~g} \mathrm{:} 19 \%$ Aktiviert $0.25 \mathrm{~g}: 14 \%$ $0.27 \mathrm{~g}: 21 \%$ 
Tabelle 2

$\mathrm{K}$-Ar-Ergebnisse an drei Biotiten der Eifel, die mit neutronenaktiviertem Material versetzt waren $\left(\mathrm{K}^{39}(\mathrm{n}, \mathrm{p}) \cdot \mathrm{Ar}^{39}\right)$.

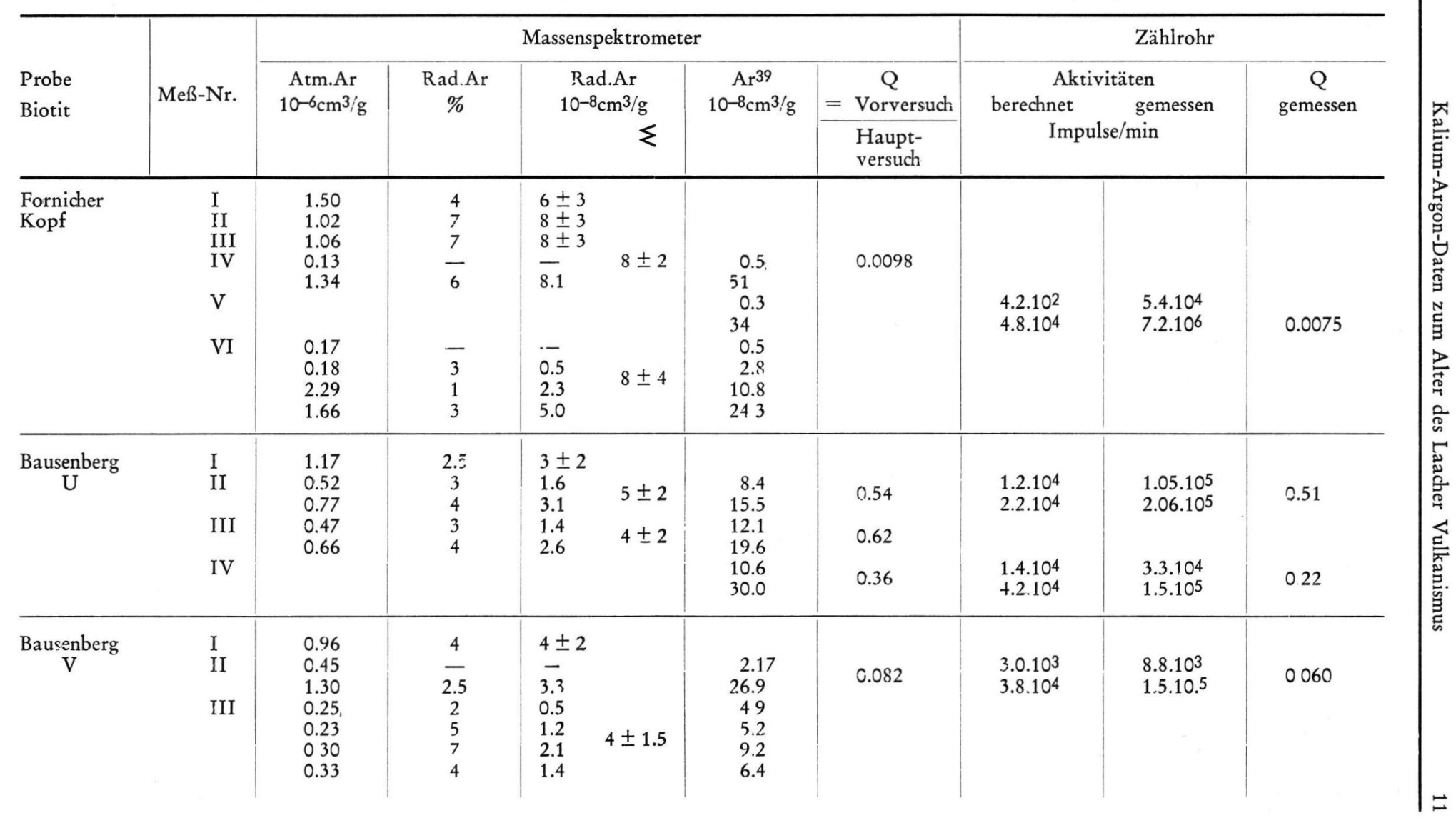


Luftargon ist zumindest in diesen Biotiten im Kristall festgehalten, weshalb die Alterszahlen nicht genauer werden können. Die Zahlen der ersten Spalten in der Tabelle 2 sind massenspektrometrisch gewonnen; die vorletzte Spalte enthält für einige dieser massenspektrometrischen Zahlen zähltechnisch gewonnene Vergleichszahlen. Leider läßt die Übereinstimmung der auf diesen verschiedenen Wegen gewonnenen Verhältnisse von Vorund Hauptversuch (Q) zu wünschen übrig. Vermutlich kommen diese Abweichungen durch die Diffusion des $\mathrm{Ar}^{37}$ zustande. $\mathrm{Da}$ dieses aus $\mathrm{Ca}$ entsteht, verhält es sich etwas anders als das $\mathrm{Ar}^{39}$. Aber es kann trotzdem behauptet werden, daß eine Korrektur auf zähltechnischem Weg die Altersangabe wesentlich verbessert, wenn die Luftargonmenge zu „hartem" Ausheizen gezwungen wird.

Zudem wurde massenspektrometrisch überprüf, ob das $\mathrm{Ar}^{39}$ wirklich proportional dem $\mathrm{Ar}^{40}$ aus dem Kristall frei wird. Etwa $20 \%$ bestrahltes Material wurde unter den Sanidin „Kärlich“ gemengt und dieses Gemisch aus bestrahltem und unbestrahltem Material stufenweise hochgeheizt. Abbildung 3 zeigt die Ergebnisse. Es ergab sich mäßige Proportionalität, welche in vielen Fällen zur Verbesserung der Datierungen beitragen könnten. Da auf gute Mischung Wert gelegt worden war, konnte eine gleichmäßige Erhitzung des bestrahlten und unbestrahlten Materials angenommen werden. Die beobachteten Abweichungen sind daher nicht zu verstehen. Es ist jedoch zu hoffen, daß in Zukunft mit diesen Hilfsmitteln bei Sanidinen oder sonstigen Mineralen, bei denen nicht erwartet werden muß, daß sowohl radiogenes wie auch atmosphärisches Argon gleichmäßig im Kristall verteilt sind, eine Verbesserung der Meßgenauigkeit bei kleinen Altern erzielt werden kann. In den meisten Fällen wird es aber bei Sanidinen und Augiten leichter sein, die Mineralkörner anzuätzen, um damit die Schichten mit dem erhöhten Luftargon zu entfernen (Evernden 1960).

\section{Ergebnisse der Datierungen}

Die Ergebnisse der Datierungen sind in den Tabellen 3 bis 6 aufgeführt. Die K-Gehalte wurden aus mindestens 2, in den meisten Fällen aus drei Messungen gemittelt. Als Maß für die Zuverlässigkeit der Datierungen kann u. a. der Anteil des atmosphärischen Argons bei der Messung angesehen werden, der deshalb in allen Fällen angegeben ist.

Die Alter sind unter Zugrundelegung der Konstanten

$$
\lambda=5.32 .10^{-10} \mathrm{a}^{-1} \text { und } \lambda / \mathrm{k} / \lambda \beta=0.123
$$

für den dualen Zerfall des Kaliums errechnet.

Die Tabelle 3 enthält die Alter von Sanidinen, die in die stratigraphische Abfolge des Laacher Vulkanismus passen, ferner die Alter von zwei Biotiten aus der Lava des Bausenbergs, die ebenfalls keine deutliche, d.h. außerhalb der Fehlergrenzen liegende Argonüberhöhung zeigen. Während die Sanidine mit sehr kleinen Fehlern meßbar waren, ergaben die Biotite ungenauere Werte, aus denen jedoch geschlossen werden kann, daß auch diese Alter in den geologischen Ablauf passen.

Tabelle 4 gibt einen Überblick über die Ergebnisse, die im Vergleich mit den stratigraphischen Gegebenheiten als zu hoch betrachtet werden müssen. Überschüsse im Argongehalt fanden sich in Sanidinen, Biotiten und Augiten. Die aus Tuffen stammenden Biotite weisen mit ca. $10^{-5} \mathrm{~cm}^{3} / \mathrm{g}$ etwa zehnmal soviel atmosphärisches Argon auf wie die Einsprenglingsbiotite aus den Basaltlaven „Bausenberg“ und „Fornicher Kopf“. In jenen ist das überschüssige Gas wahrscheinlich durch den guten Kontakt mit der Atmophäre angereichert worden.

Mit Überschüssen bei den Augitproben war nach den Ergebnissen von HART \& DoDD (1962) zu rechnen, die darauf hinwiesen, daß dadurch gerade bei jungen Augiten große Fehler entstehen können.

Zum Vergleich wurde ein Augit vom Aetna analysiert, der von Professor P. BicheT gesammelt und von Professor LabeYrie überlassen worden war. An diesem rezenten Augit 
Tabelle 3

K-Ar-Alter pleistozäner Sanidine und Biotite der Eifel.

\begin{tabular}{|c|c|c|c|c|}
\hline Probe & $\begin{array}{c}\operatorname{Ar}(\mathrm{rad}) \\
10^{-7} \mathrm{~cm}^{3} / \mathrm{g}\end{array}$ & $\begin{array}{c}(\mathrm{atm}) \\
\%\end{array}$ & $\begin{array}{l}\mathrm{K} \\
\partial \%\end{array}$ & $\begin{array}{l}\text { Alter } \\
106 \mathrm{a}\end{array}$ \\
\hline \multicolumn{5}{|l|}{ Sanidine: } \\
\hline $\begin{array}{l}\text { Hohenfels } \\
\text { (Mühlenberg) }\end{array}$ & 1.76 & 18 & 9.80 & $0.46 \pm 0.02$ \\
\hline $\begin{array}{l}\text { Rockes-Kyll } \\
\text { (Kyller Kopf) }\end{array}$ & 1.65 & 23 & 9.83 & $0.43 \pm 0.02$ \\
\hline Rieden & 1.83 & 77 & 10.9 & $0.42 \pm 0.03$ \\
\hline Leilenkopf I & 1.71 & 10 & 10.7 & $0.40 \pm 0.02$ \\
\hline II & 1.68 & 24 & 10.8 & $0.39 \pm 0.02$ \\
\hline II & 1.66 & 27 & 10.8 & $0.39 \pm 0.02$ \\
\hline Selbergit-Tuff I & 1.75 & 19 & 12.5 & $0.35 \pm 0.02$ \\
\hline (Hoheley) II & 1.65 & 17 & 12.2 & $0.35 \pm 0.02$ \\
\hline $\begin{array}{l}\text { Selbergit-Tuff } \\
\text { „In der Erle“ }\end{array}$ & 1.53 & 28 & 11.1 & $0.35 \pm 0.02$ \\
\hline $\begin{array}{l}\text { Biotite: } \\
\text { Bausenberg I }\end{array}$ & 0.4 & 96 & 5.1 & $0.2 \pm 0.1$ \\
\hline II & 0.4 & 96 & 5.2 & $0.2 \pm 0.1$ \\
\hline
\end{tabular}

Tabelle 4

Überschüssiges radiogenes Argon in Mineralen der Eifel.

\begin{tabular}{|c|c|c|c|c|}
\hline Probe & $\begin{array}{c}\operatorname{Ar}(\mathrm{rad}) \\
10^{-7} \mathrm{~cm}^{3 / g} \mathrm{~g}\end{array}$ & $\begin{array}{c}(\mathrm{atm}) \\
\%\end{array}$ & $\begin{array}{l}\mathrm{K} \\
\%\end{array}$ & $\begin{array}{l}\text { Alter } \\
106 \mathrm{a}\end{array}$ \\
\hline $\begin{array}{l}\text { S a nidine: } \\
\text { Leubsdorf }\end{array}$ & 2.48 & 22 & 10.0 & $0.63 \pm 0.03$ \\
\hline Kärlich & 5.68 & 13 & 5.66 & $2.5,4 \pm 0.15$ \\
\hline Laach I & 0.36 & 72 & 4.00 & $0.23 \pm 0.03$ \\
\hline II & 3.54 & 22 & 5.90 & $1.51 \pm 0.10$ \\
\hline $\begin{array}{l}\text { B i o t i t e : } \\
\text { Fornicher Kopf }\end{array}$ & 0.8 & 93 & 72 & $0.28 \pm 0.10$ \\
\hline Leilenkopf II & 4.0 & 96 & 7.6 & $1.3 \pm 0.4$ \\
\hline Hoheley & 4.4 & 94 & 4.5 & $2.5 \pm 0.8$ \\
\hline Thürer Wald & 4.2 & 95 & 7.1 & $1.5 \pm 0.4$ \\
\hline Leilenkopf III & 4.3 & 92 & 7.3 & $1.5 \pm 0.3$ \\
\hline $\begin{array}{l}\text { A ugite : } \\
\text { Fornicher Kopf }\end{array}$ & 0.29 & 93 & 0.21 & $3.5 \pm 1$ \\
\hline Bausenberg & 1.12 & 56 & 0.26 & $11 \pm 1$ \\
\hline $\begin{array}{l}\text { Zum Vergleich: } \\
\text { Augit Atna }\end{array}$ & $<0.06$ & $>98$ & - & $<0.5$ \\
\hline Augit Vias & 1.0 & 50 & 0.02 & $>160$ \\
\hline
\end{tabular}


Tabelle 5

K-Ar-Alter und Altersabschätzungen von Gesteinen der Eifel.

\begin{tabular}{l|c|c|c|c}
\hline Probe & $\begin{array}{c}\mathrm{Ar}(\mathrm{rad}) \\
10^{-7} \mathrm{~cm}^{3} / \mathrm{g}\end{array}$ & $\begin{array}{c}(\mathrm{atm}) \\
\%\end{array}$ & $\begin{array}{c}\mathrm{K} \\
\%\end{array}$ & $\begin{array}{c}\text { Alter } \\
106 \mathrm{a}\end{array}$ \\
\hline Schellkopf & $1.74 \pm 0.06$ & 46 & 7.75 & $0.57 \pm 0.04$ \\
Olbrück & $1.19 \pm 0.06$ & 54 & 7.27 & $0.41 \pm 0.03$ \\
Sulzbusch & $0.19 \pm 0.04$ & 93 & 1.43 & $0.34 \pm 0.08$ \\
Perler Kopf & $0.86 \pm 0.07$ & 72 & 6.70 & $0.32 \pm 0.03$ \\
Engelner Kopf & $0.53 \pm 0.05$ & 88 & 4.22 & $0.32 \pm 0.04$ \\
Hochsimmer & $0.5 \pm 0.15$ & 95 & 3.8 & $0.3 \pm 0.1$ \\
Hochstein & $0.15 \pm 0.04$ & 90 & 1.49 & $0.26 \pm 0.08$ \\
Leilenkopf & $0.22 \pm 0.03$ & 92 & 2.50 & $0.22 \pm 0.04$ \\
Thürer Wald & $0.21 \pm 0.03$ & 84 & 3.04 & $0.18 \pm 0.03$ \\
(Nettetal) & $0.18 \pm 0.04$ & 93 & 2.97 & $0.15 \pm 0.04$ \\
Bausenberg I & $0.15 \pm 0.03$ & 91 & 2.63 & $0.14 \pm 0.03$ \\
$\quad$ II & $0.11 \pm 0.04$ & 94 & 2.64 & $0.10 \pm 0.04$ \\
Mauerley & $0.10 \pm 0.04$ & 91 & 2.74 & $0.09 \pm 0.04$ \\
Lummerfeld & $0.30 \pm 0.04$ & 90 & 2.80 & $0.27 \pm 0.04$ \\
Fornicher Kopf & & & &
\end{tabular}

Ta be 11 e 6

K-Ar-Alter pliozäner und pleistozäner Gesteine von Hérault, Frankreich.

\begin{tabular}{l|c|c|c|c|c}
\hline Vorkommen & $\begin{array}{c}\text { Kalium } \\
\%\end{array}$ & $\begin{array}{c}\operatorname{Ar}(\mathrm{rad}) \\
10-7 \mathrm{cc} / \mathrm{g}\end{array}$ & $\begin{array}{c}\operatorname{Ar}(\mathrm{atm}) \\
\%\end{array}$ & $\begin{array}{c}\text { K-Ar-Alter } \\
106 \mathrm{a}\end{array}$ & Geol. Alter \\
\hline I H é r a ult t & & & & & \\
Roque Haute & 0.87 & 0.22 & 87 & $0.64 \pm 0.09$ & \\
Vias & 1.10 & 0.29 & 81 & $0.67 \pm 0.05$ & Pleistozän \\
St. Thibery & 1.29 & 0.35 & 78 & $0.68 \pm 0.06$ & \\
Agde & 1.29 & 0.37 & 78 & $0.73 \pm 0.07$ & Pleistozän \\
Agde & 1.16 & 0.34 & 82 & $0.74 \pm 0.07$ & Villafranchien \\
Valros & 0.65 & 0.37 & 80 & $1.4 \pm 0.1$ & Villafranchien \\
II O ld u va i & 0.92 & 0.63 & 92 & $1.7 \pm 0.3$ &
\end{tabular}

konnte kein Überschuß von Argon festgestellt werden. Dagegen wies ein Augit von Agde (Fundpunkt Vias) einen beträchtlichen Überschuß auf.

Tabelle 5 enthält die an Gestein gewonnenen Alterswerte. Einige Messungen sind infolge des relativ hohen Gehaltes an atmosphärischem Argon mit einer größeren Ungenauigkeit behaftet. Schwierigkeiten infolge ererbten Argons traten nur an der Probe "Fornicher Kopf“ auf. Die Werte der Proben „Mauerley“ und „Burgbrohl“ sind zu niedrig. Bei allen anderen Gesteinen fügen sich die Alter gut in die Stratigraphie ein.

Tabelle 6 enthält die Alter einiger Lavavorkommen bei Agde, Dept. Hérault, in Südfrankreich. Sie wurden bestimmt, um ergänzende Daten über die Länge des Pleistozäns zu erhalten. Die Meßfehler innerhalb dieser Serie sind infolge apparativer Schwierigkeiten größer als in den Eifel-Messungen.

Die Datierung Olduvai IV ist eine Neudatierung. Sie wurde an einem Gestein durchgeführt, das Herr Professor Heberer (Göttingen) zur Verfügung stellte. Es war wesentlich frischer als die vorher datierten Proben (v. Koenigswald \& a., 1962), enthielt aber viel atmosphärisches Argon, was das Alter wahrscheinlich beeinflußt hat. 
Tabe 11 e 7

Chemische Zusammensetzung der für K-Ar-Bestimmungen benutzten Gesteine des Laacher Vulkangebietes.

\begin{tabular}{|c|c|c|c|c|c|c|c|c|}
\hline & Schellkopf & Olbrück & $\begin{array}{c}\text { Engelner } \\
\text { Kopf }\end{array}$ & Perler Kopf & Sulzbusch & Hochsimmer & Hochstein & Bausenberg \\
\hline $\mathrm{SiO}_{2}$ & 50.41 & 52.51 & 50.09 & 46.37 & 44.30 & 43.40 & 44.00 & 41.60 \\
\hline $\mathrm{TiO}_{2}$ & 0.40 & 0.32 & - & 0.98 & 2.62 & 1.79 & 1.40 & 3.20 \\
\hline $\mathrm{Al}_{2} \mathrm{O}_{3}$ & 22.15 & 21.37 & 23.96 & 17.27 & 15.05 & 16.07 & 14.40 & 15.12 \\
\hline $\mathrm{Fe}_{2} \mathrm{O}_{3}$ & 2.27 & 2.15 & 2.22 & 4.05 & 5.00 & 6.64 & 4.70 & 4.78 \\
\hline $\mathrm{FeO}$ & 1.12 & 1.04 & 0.06 & 3.04 & 5.75 & 3.53 & 4.77 & 4.67 \\
\hline $\mathrm{MnO}$ & 0.23 & 0.55 & - & 0.28 & 0.51 & 1.35 & 0.20 & - \\
\hline $\mathrm{MgO}$ & 0.16 & 0.11 & 0.28 & 1.29 & 5.57 & 4.63 & 9.75 & 10.01 \\
\hline $\mathrm{CaO}$ & 1.75 & 2.16 & 1.81 & 7.83 & 10.37 & 10.32 & 11.05 & 12.80 \\
\hline $\mathrm{BaO}$ & - & 0.06 & - & - & - & - & 0.13 & - \\
\hline $\mathrm{Na}_{\varepsilon} \mathrm{O}$ & 8.83 & 7.21 & 8.05 & 8.04 & 5.67 & 5.81 & 4.35 & 3.03 \\
\hline $\mathrm{K}_{2} \mathrm{O}$ & 9.10 & 8.97 & 9.28 & 6.29 & 4.35 & 4.93 & 3.23 & 3.39 \\
\hline $\mathrm{CO}_{2}$ & 0.31 & n.b. & 0.22 & 1.18 & 0.38 & - & - & 0.01 \\
\hline $\mathrm{Cl}$ & 0.38 & 0.56 & - & 0.34 & 0.11 & 0.19 & 0.08 & - \\
\hline $\mathrm{SO}_{3}$ & 0.61 & 0.62 & 0.82 & 1.03 & 0.03 & 0.41 & 0.14 & - \\
\hline $\mathrm{P}_{2} \mathrm{O}_{5}$ & - & n.b. & 0.05 & 0.25 & 0.45 & 0.78 & 0.49 & 0.26 \\
\hline F & - & 0.02 & - & - & - & - & - & - \\
\hline $\mathrm{H}_{2} \mathrm{O}+$ & 2.65 & 1.95 & 2.12 & 1.50 & 0.63 & 0.48 & 0.40 & 0.82 \\
\hline \multirow[t]{2}{*}{$\mathrm{H}_{2} \mathrm{O}-$} & - & 0.75 & 1.15 & 0.54 & 0.11 & 0.20 & 0.55 & - \\
\hline & 100.37 & 100.35 & 100.11 & 100.28 & 100.88 & 100.53 & 99.64 & 99.69 \\
\hline
\end{tabular}


Tabelle 8

Mineralbestand der für K-Ar-Bestimmungen benutzten Gesteine des Laacher Vulkangebietes.

\begin{tabular}{|c|c|c|c|c|c|c|c|c|}
\hline & Schellkopf & Olbrück & Engelner Kopf & Perler Kopf & Sulzbusch & Hochsimmer & Hochstein & Bausenberg \\
\hline Sanidin & $27.9(\mathrm{ab} \mathrm{26})$ & $36.9(\mathrm{ab} 44)$ & $22.6(\mathrm{ab} 29)$ & $5.8(a b 50)$ & - & - & - & - \\
\hline Plagioklas & - & - & - & - & - & $\mathrm{Sp}$ & 10.8 & $7.5($ an 55$)$ \\
\hline Leucit & $26.0(\mathrm{Na}-\mathrm{Le} 5)$ & 26.6(Na-Le5) & $24.9(\mathrm{Na}-\mathrm{Le} 4)$ & $32.6(\mathrm{Na}-\mathrm{Le} 5)$ & 28.8 & $27.7(\mathrm{Na}-\mathrm{Le} 5)$ & 16.0 & $17.8(\mathrm{Na}-\mathrm{Le} 5)$ \\
\hline Nephelin & $21.6(\mathrm{ks} 9)$ & $10.5(\mathrm{Ks} 22)$ & $30.5(\mathrm{ks} 18)$ & $19.0(\mathrm{ks} 0)$ & 21.3 & $20.7(\mathrm{ks} \mathrm{3})$ & 14.6 & $14.4(\mathrm{ks} 15)$ \\
\hline Nosean & 9.4 & 9.0 & 12.1 & - & - & - & - & - \\
\hline Hauyn & - & - & - & 8.1 & - & 3.6 & - & - \\
\hline Sodalith & 5.3 & 7.8 & - & 5.3 & - & 1.2 & 2.2 & - \\
\hline Biotit & $\mathrm{Sp}$ & - & $\mathrm{Sp}$ & - & 1.2 & - & - & $\mathrm{Sp}$ \\
\hline Augit & 8.4 & 6.4 & 8.7 & 23.0 & 42.6 & 40.8 & 45.2 & 46.2 \\
\hline Olivin & - & - & $\mathrm{Sp}$ & - & 0.5 & $\mathrm{Sp}$ & 7.6 & $7.8(\mathrm{fa} 10)$ \\
\hline Melanit & - & - & - & 1.5 & - & - & - & - \\
\hline Magnetit & $\mathrm{Sp}$ & 0.5 & $\mathrm{Sp}$ & - & 3.8 & 4.2 & 2.4 & 5.7 \\
\hline Titanit & 0.7 & 0.6 & 0.6 & 1.2 & - & - & - & - \\
\hline Apatit & - & $\mathrm{Sp}$ & 0.1 & 0.5 & 1.2 & 1.8 & 1.2 & 0.6 \\
\hline \multirow[t]{2}{*}{ Calcit } & 0.7 & 1.8 & 0.5 & 3.0 & 0.7 & - & - & - \\
\hline & 100.0 & 100.0 & 100.0 & 100.0 & 100.0 & 100.00 & 100.0 & 100.0 \\
\hline
\end{tabular}




\section{Möglichkeiten der stratigraphischen Einordnung der K-Ar-Daten aus dem Laacher-See-Gebiet}

Den wichtigsten Anhalt für die stratigraphische Einordnung der an Mineralen und Gesteinen aus dem Laacher-See-Gebiet gewonnenen K-Ar-Daten geben die Schotterauflagerungen der pleistozänen Flußterrassen im Mittelrheingebiet. Die Terrassen werden hier nach der von J. I. S. ZonNeveld (in: Woldstedt 1958) aufgestellten Gliederung unterschieden und benannt (Abb. 4). Die Höhenangaben beziehen sich auf die Auflagerungsflächen der Schotterdecken auf den Felsverebnungen oder Felsterrassen.

Sedimentpetrographische Untersuchungen der Terrassenschotter in dem an das LaacherSee-Gebiet angrenzenden Teil des Mittelrheintales (Frechen \& v. D. Bоoм 1959) ergaben, $\mathrm{da}$ in ihnen von der älteren Hauptterrasse bis zur jüngeren Niederterrasse in geringerer oder größerer Menge Bestandteile auftreten, die aus den Förderungen der Laacher Vulkane, insbesondere den explosiv ausgeworfenen Lockermassen, stammen. Sie gelangten in

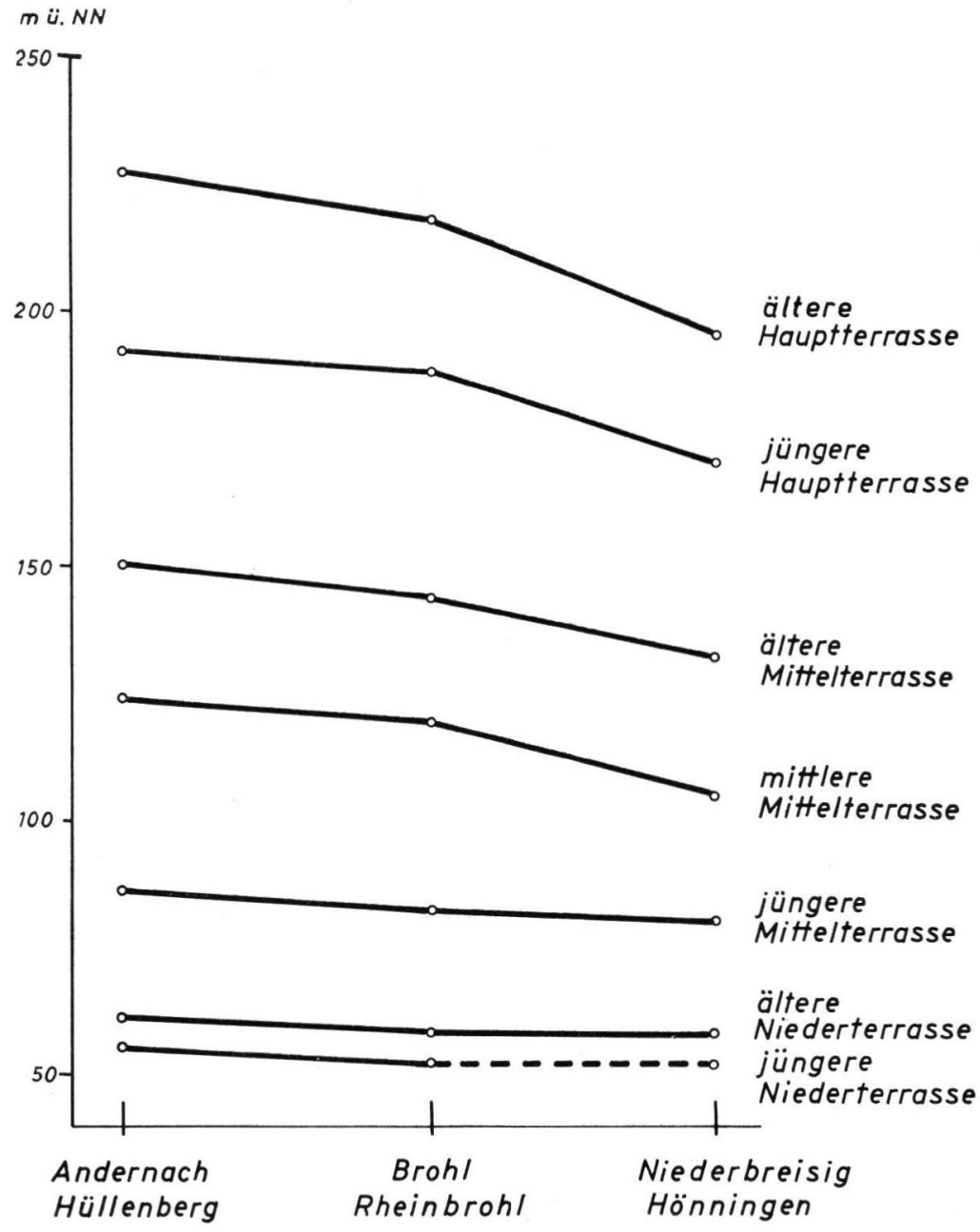

Abb. 4. Höhenlage der Schotterterrassen (Auflagerungsflächen) des Rheines zwischen Andernach Niederbreisig, nach J. I. S. ZONNEveLD, aus P. WoldSTEDT 1958. 
den einzelnen Tätigkeitsphasen nach kurzem Wassertransport, durch Windverwehung oder auch unmittelbar vulkanisch-äolisch in verhältnismäßig kurzer Zeit in die Sedimente des Rheines. Während der langen Vulkantätigkeit hat sich die Zusammensetzung der Magmen mehrfach geändert. Hierauf ist es zurückzuführen, daß die Schotter der einzelnen Terrassen unterschiedliche vulkanische Minerale und Gesteine enthalten, die sich petrographisch mit bestimmten Förderstellen im Laacher-See-Gebiet in Verbindung bringen lassen. Dadurch ist es möglich, in Anlehnung an die Terrassengliederung eine relative Altersfolge der vulkanischen Vorgänge aufzustellen.

Die Schotter der älteren und jüngeren Hauptterrasse enthalten vor allem Minerale aus den Selbergittuffen im nordwestlichen Teil des Laacher-See-Gebietes. Hauptschauplatz der Vulktantätigkeit war der Riedener Kessel, in dem die Ausbrüche zuletzt einen stark-explosiven Charakter annahmen.

Nach der Hauptförderphase der Selbergittuffe und der mit ihnen auftretenden Selbergitgesteine sind vor und während der Bildungszeit der verschiedenen Mittelterrassen im wesentlichen alkalibasaltische Schmelzen aufgedrungen, deren Bestandteile nun in den Schottern vorherrschen.

Bimssteineinlagerungen in den Schottern der Niederterrasse zeigen eine neue Phase explosiver Tätigkeit an, die zu mehrfachen Ausbrüchen im Wehrer Kessel führte und im Alleröd mit dem großen Bimssteinausbruch im Laacher Kessel ihr Ende fand. Außerdem erfolgten, besonders im östlichen Teil des Laacher-See-Gebietes, auch umfangreiche Förderungen alkalibasaltischer Tuffe und Laven.

Durch die Ergebnisse der sedimentpetrographischen Untersuchungen sind die Hauptphasen der Vulkantätigkeit im Laacher-See-Gebiet festgelegt. Die stratigraphische Einordnung der K-Ar-Daten erfordert aber, daß die Vulkanausbrüche, durch welche die für die Bestimmungen verwendeten Minerale und Gesteine an die Oberfläche kamen, im einzelnen genauer mit den Aufschotterungen der Rheinterrassen in Verbindung gebracht werden.

Die Korrelierung einzelner vulkanischer Vorgänge mit bestimmten Aufschotterungsphasen läßt sich am sichersten durchführen, wenn die geförderten magmatischen Stoffe als Tuffe unmittelbar äolisch in die Terrassensedimente abgelagert wurden. Bei diesen primärvulkanischen Einschaltungen handelt es sich um Bestandteile, die durch heftige Gasausbrüche weithin gestreut worden sind. Die Horizonte, in denen sie auftreten, bilden wichtige Fixpunkte für die Datierung des Vulkanismus und der Terrassen, weil das vulkanische Ereignis und die Einstreuung des Lockermaterials in die Schotter als gleichzeitig angesehen werden können. Primär-vulkanisch abgelagerte Tuffe finden sich in den Schottern der jüngeren Hauptterrasse, der mittlereh Mittelterrasse und der älteren Niederterrasse.

Eine weitere Möglichkeit der stratigraphischen Festlegung von vulkanischen Bildungen ergibt sich aus ihren Lagebeziehungen zu den Flußterrassen. Tuffe oder Lavaströme, die einer Terrasse aufliegen, sind jünger als diese und wenn sie bei der weiteren Taleintiefung noch angeschnitten wurden, kann ihre Entstehung zudem in die Zeit vor der Bildung der nächsttieferen Terrasse verlegt werden. Auf diese Weise läßt sich ein Zeitintervall angeben, in dem der vulkanische Vorgang stattgefunden hat. Der Spielraum für die Einordnung der an solchen Vorkommen bestimmten K-Ar-Daten ist naturgemäß größer als im Falle einer direkten Einschaltung von Tuff in Terrassenschotter.

Es wird jetzt meist angenommen, daß die Aufschotterung der Flußterrassen jeweils in Kaltzeiten stattgefunden hat (P. Woldstedt 1961). Recht sichere Anhaltspunkte für die Zuordnung der K-Ar-Daten können daher auch Klimaindikatoren geben, die an zahlreichen Stellen in den Tuffen des Laacher Vulkangebietes zu beobachten sind. So finden sich in dem primär gelagerten Bimssteintuff der Alleröd-Warmzeit Negativformen von Baumstämmen, Ästen und Zweigen, in den in der Kaltphase der jüngeren Tundrenzeit umgelagerten Bimssteintuffen Kryturbations- und Verwehungsstrukturen. In Analogie zu 
diesen Erscheinungen wurden Baumnegative und Pflanzenabdrücke in älteren Tuffen als Hinweise auf Warmzeiten, Kryoturbations- und Verwehungsstrukturen als Hinweise auf Kaltzeiten gewertet. In manchen Fällen sind Kaltzeiten auch durch Einlagerungen von Löß markiert.

\section{Beziehung der K-Ar-Daten zu den Flußterrassen im Mittelrheingebiet}

Selbergit des Schellkopf bei Brenk

Alter: $570.10^{3} \mathrm{a}$

Der Selbergit vom Schellkopf ergab das höchste bisher im Laacher Vulkangebiet bestimmte Alter.

Oberhalb von Fornich bei Brohl treten in den Schottern der älteren Hauptterrasse des Rheines bereits an der Basis größere Kristalle von Ägirinaugit, barkevikitischer Hornblende und frischem Biotit auf, die nur schwache Rundungserscheinungen zeigen. Man kann annehmen, daß die Minerale von einem nicht weit entfernten, schon vor der Aufschotterung der älteren Hauptterrasse tätigen Vulkan stammen.

Der Grenztuff des Schellkopfs enthält neben Nosean und Sanidin Kristalle von Ägirinaugit, barkevikitischer Hornblende und Biotit, die zum Teil cm-Größe erreichen. $\mathrm{Da}$ die übrigen Vorkommen von Selbergit und Selbergittuff im Laacher-See-Gebiet alle wesentlich jünger sind (Abb. 8), wird man die vulkanischen Minerale in den Schottern der älteren Hauptterrasse aus dem Tuff des Schellkopfs herleiten können.

Nachdem durch die übrigen Daten der Abb. 8 die Größenordnung von Glacial- und Interglacialzeiten abgeschätzt werden konnte (siehe Abschnitt 9), wurde das Alter des Schellkopfs in den Zeitabschnitt des Pleistozäns vor der Waal-Warmzeit gestellt. Die hier befindlichen Daten lassen sich vorerst noch nicht bestimmten Kalt- und Warmzeiten zuordnen. Aus dem Alterswert „Schellkopf“ kann aber gefolgert werden, daß die Schotter der älteren Hauptterrasse jünger als $570.10^{3}$ a sind.

Sanidine vom Leilenkopf bei Niederlützingen

Alter: "Leilenkopf I“ $405.10^{3} \mathrm{a}$

"Leilenkopf II“ $390.10^{3} \mathrm{a}$

$390.10^{3} \mathrm{a}$

Der alkalibasaltische Schichtvulkan Leilenkopf ist in der noch erhaltenen nördlichen Hälfte fast bis auf das Niveau der älteren Hauptterrasse erniedrigt. Seine südliche Hälfte wurde während der Eintiefung des Brohltales vollständig zerstört und entfernt.

Nach der Ausbildung und Lagerungsweise lassen sich am Leilenkopf drei Tuffarten unterscheiden:

Der Tuff "Leilenkopf I“ besteht aus geschichteten und etwas verschweißten Wurfschlacken. Er bildet den äußeren Vulkanwall. Ein Sanidin aus diesem Tuff ergab das Alter 405.103a.

Der Tuff „Leilenkopf II“, der im Zentrum des Vulkanes auftritt, besteht überwiegend aus unregelmäßig gelagerten fester verschweißten Lapilli. Er läßt an vielen Stellen eine starke Durcharbeitung erkennen, wie sie für Schlotfüllungen oft charakteristisch ist. An zwei Sanidinen aus dem Lapillituff wurden die Alter $390.10^{3}$ a bestimmt.

Der jüngste Tuff „Leilenkopf III“ ist durch einen Lößhorizont von den Tuffen I und II getrennt. Er wird später behandelt.

Die zur Altersbestimmung verwendeten Sanidine finden sich als Auswürflinge in den älteren Tuffen I und II des Leilenkopf-Vulkanes. Sie gehören genetisch zu Foyait-Pegmatiten, die in der Tiefe vom Leilenkopf-Magma durchbrochen und aufgenommen wurden.

Die Wurfschlacken der Eruption „Leilenkopf I“ lagern nach Nordosten auf Schottern der älteren Hauptterrasse. Zwischen den Schottern und den Schlacken befindet sich an 
einigen Stellen ungestörter Löß von 2-3 m Mächtigkeit. Die Schotter streichen an der Oberkante der steilen Hänge des Brohltales mit $20-25^{\circ}$ Neigung nach oben in die Luft aus. Der tiefere Eruptionstrichter, der mit dem Tuff „Leilenkopf II“ gefüllt ist, wurde bei der Taleintiefung angeschnitten.

Die Lagerungsweise der am Talrand noch erhaltenen Reste des Vulkanes ist nur verständlich, wenn man annimmt, daß seine sedimentäre Unterlage sich zur Zeit der älteren Ausbrüche noch weiter nach Süden in den Raum des jetzigen Brohltales ausdehnte. Dies bedeutet, daß das Brohltal noch nicht unter die an der Talkante anstehenden Schotter der älteren Hauptterrasse eingetieft und demnach an dieser Stelle noch nicht vorhanden war.

Nach dem Alter „Leilenkopf I“ sind die Schotter der älteren Hauptterrasse älter als 405.103a. Die Eintiefung des Brohltales begann nach dieser Zeit. Man kann annehmen, daß sich die Entstehung des Rheintales, das die Erosionsbasis für das Brohltal bildete, an der Einmündung des Brohltales, wo sich der Leilenkopf befindet, vollkommen synchron vollzogen hat. Die ältere Hauptterrasse des Rheines wäre demnach ebenfalls älter als $405.10^{3} \mathrm{a}$ und die jüngere Hauptterrasse jünger als $405.10^{3} \mathrm{a}$.

Der Lapillituff „Leilenkopf II“ in den tieferen Teilen des Trichteranschnittes ist entweder jünger als der Tuff „Leilenkopf I“ oder im Schlot noch längere Zeit durch heiße Gase erhitzt worden. Mit den Schottern der älteren Hauptterrasse steht er nicht in unmittelbarer Berührung. Die Alter „Leilenkopf II“ 390 und 390.103a können daher für die Datierung des Beginnes der Taleintiefung nicht direkt benutzt werden.

Wenn man die Aufschotterung der älteren Hauptterrasse in eine Kaltzeit und die danach beginnende Eintiefung des Brohltales in eine Warmzeit verlegt, ist der Tuff „Leilenkopf I“ ungefähr in den Übergang zwischen einer Kaltzeit und der darauf folgenden Warmzeit zu verlegen.

Selbergit der Olbrück bei Hain, Brohltal

Alter: $410.10^{3} \mathrm{a}$

Der an das Brohltal stoßende Selbergit der Olbrück, der zum größten Teil in devonischem Sedimentgestein steht und die alte Einebnungsfläche des Schiefergebirges etwas überragt, ist durch Erosion in der vollen Höhe des Talhanges angeschnitten worden. Da er nach seinem Auftreten als Quellkuppe auch an der talwärtigen Seite in ein vorher vorhandenes Gestein intrudiert sein muß, kann zur Zeit seiner Entstehung das Brohltal an dieser Stelle noch nicht vorhanden gewesen sein (AHrens 1930). Das Alter der Olbrück gibt damit einen weiteren Anhaltspunkt für die zeitliche Festlegung der Talentwicklung im Mittelrheingebiet.

Aus den Zusammenhängen am Leilenkopf und dem Alter „Leilenkopf I“ war gefolgert worden, daß der Beginn der Eintiefung der Steiltäler des Rheines und seiner Nebenflüsse jünger als $405.10^{3} \mathrm{a}$ ist. Diese Annahme wird gestützt durch das Alter der Olbrück, nach dem die Eintiefung nach $410.10^{3}$ a begann.

Sanidine aus dem Selbergit von Rieden und dem selbergitischen Bimssteintuff der Gruben "Hoheley" und "In der Erle“

Alter: Sanidin aus Selbergit $420.13^{3} \mathrm{a}$

$$
\begin{array}{ll}
\text { Sanidine "Hoheley“ } & 350.10^{3} \mathrm{a} \\
& 350.10^{3} \mathrm{a} \\
\text { Sanidin "In der Erle“ } & 350.10^{3} \mathrm{a}
\end{array}
$$

Die Gesamtablagerung der Selbergittuffe im Riedener Kessel läßt sich gliedern in den unteren blockreichen Basistuff, den mittleren, größtenteils feinkörnigen und geschichteten Tuff und den oberen Bimssteintuff (Abb. 5).

Der Basistuff wird an der Hardt bei Rieden discordant vom mittleren Tuff überlagert. Der obere Bimssteintuff enthält als Auswürflinge Stücke von schon verfestigtem mittlerem Tuff. 


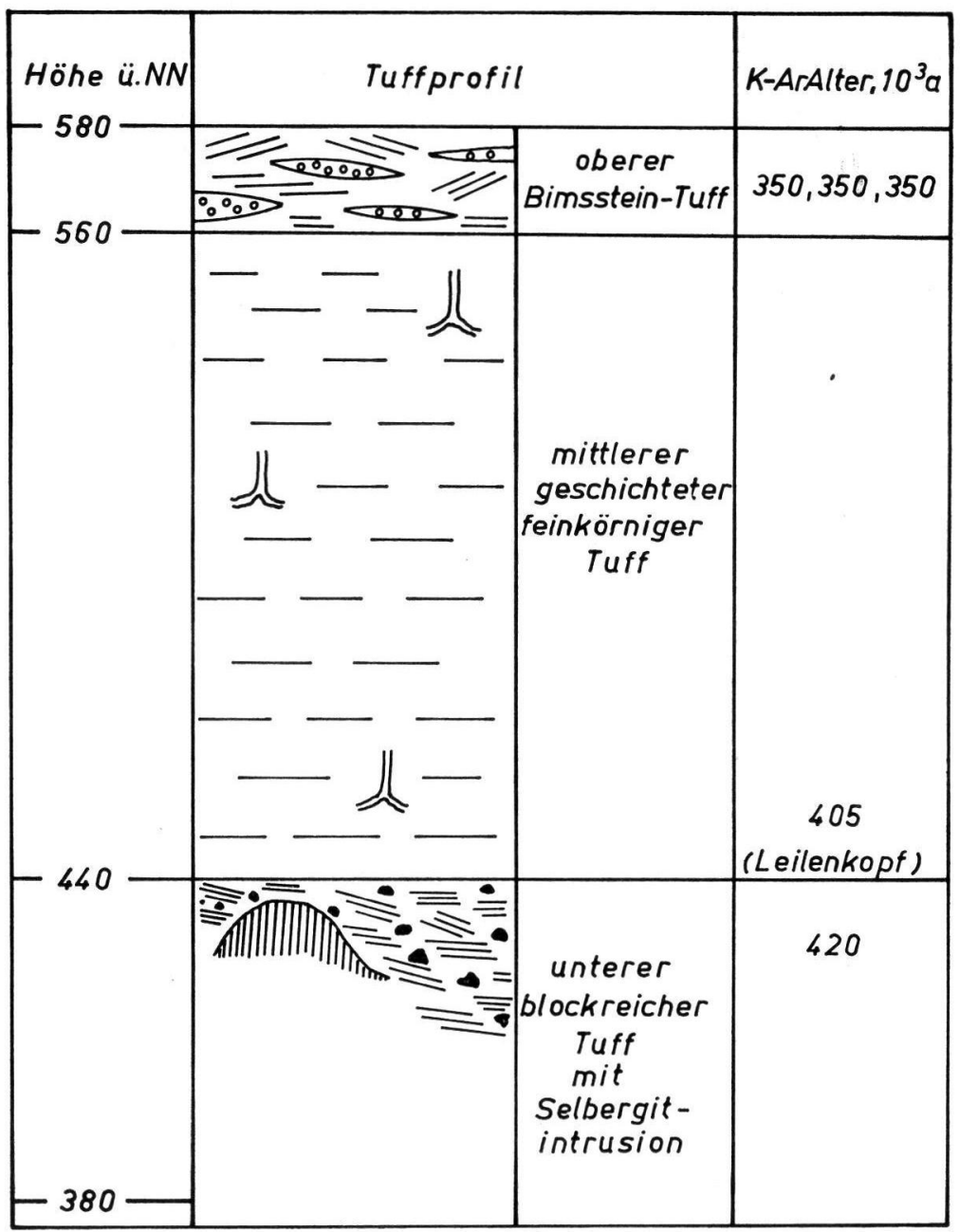

Abb. 5. Profil der Selbergit-Tuffe im Riedener Kessel, Laacher Vulkangebiet.

Im Basistuff treten Verwehungsstrukturen auf, die als Hinweis auf eine Kaltzeit angesehen werden. Der mittlere Tuff enthält Blattabdrücke, Holzeinschlüsse und Negativformen von Baumstämmen, die Zeugen einer Warmzeit darstellen. Der darüber folgende Bimssteintuff weist wieder starke Verwehungsstrukturen auf, die eine neue Kaltzeit anzeigen.

Eine analoge Abfolge von Klimaindikatoren läßt sich im Profil der Selbergittuffe in der Umgebung der Rodderhöfe bei Ettringen beobachten, Verwehungen an der Basis, Baumnegative im mittleren Tuff und Verwehungen im oberen Bimssteintuff.

Ein Sanidin aus dem im Zusammenhang mit der Förderung des Basistuffes aufgedrungenen Selbergit von der Hardt bei Rieden ergab das Alter 420.103a. Zwei Sanidine aus der Grube „Hoheley" und ein Sanidin aus der Grube „In der Erle“, die alle aus dem oberen Bimssteintuff stammen, besitzen die Alter 350.103a.

Die Zeitspanne zwischen den Altern der in den beiden Kaltzeiten geförderten Sanidine beträgt ungefähr 70.103a. Die Daten gehören nach diesem zeitlichen Abstand in zwei ver- 
schiedene Glaciale, die durch ein Interglacial, in dem der mittlere Tuff abgelagert wurde, getrennt sind. Nach den Altern „Olbrück“ und „Leilenkopf I“, die an der Wende Kaltzeit der älteren Hauptterrasse zur Warmzeit zwischen der älteren und jüngeren Hauptterrasse stehen, kann das erste Glacial im Tuffprofil des Riedener Kessels gleichgesetzt werden mit der Kaltzeit, in der die ältere Hauptterrasse, das zweite mit der Kaltzeit, in der die jüngere Hauptterrasse aufgeschottert wurde.

In den Schottern der jüngeren Hauptterrasse auf der Kettiger Höhe bei Kärlich (Neuwieder Becken), die von der Basis an basaltischen Augit und Ägirinaugit aus den wechsellagernden Basalt- und Selbergittuffen im Raume des Riedener Kessels führen, nimmt die Menge das Ägirinaugit in der oberen Hälfte des Profiles sprunghaft zu, während der Anteil der basaltischen Augite sich nur wenig ändert (Abb. 6). Das vermehrte Auftreten von Ägirinaugit wird mit dem Ausbruch des oberen selbergitischen Bimssteintuffes im Riedener Kessel in Zusammenhang gebracht, der in einer Kaltzeit stattfand, die nach den Sanidinaltern jünger ist als die Kaltzeit der älteren Hauptterrasse. Der Bimsstein ist durch starke Ausbrüche wahrscheinlich zum Teil primäräolisch, zum Teil unter den glacialen Klimabedingungen auch durch Verwehung in den Schottern der jüngeren Hauptterrasse zur Ablagerung gekommen.

Der obere Teil der Schotter der jüngeren Hauptterrasse besitzt danach ein Alter von ungefähr 350.103a. Das Alter 420.103a fällt in die Zeit der Aufschotterung der älteren Hauptterrasse.

Alkalibasalt-Lavades Sulzbusch

Alter: $340.10^{3} \mathrm{a}$

Die Lockermassen des Sulzbusch lagern gegen das Nettetal überall auf devonischem Untergrund. An der Nordwestflanke ist der Berg von unten an von Selbergittuff bedeckt. Der Hauptkegel ist zum Teil älter als die Selbergittuffe an den Rodderhöfen. Vom öst-
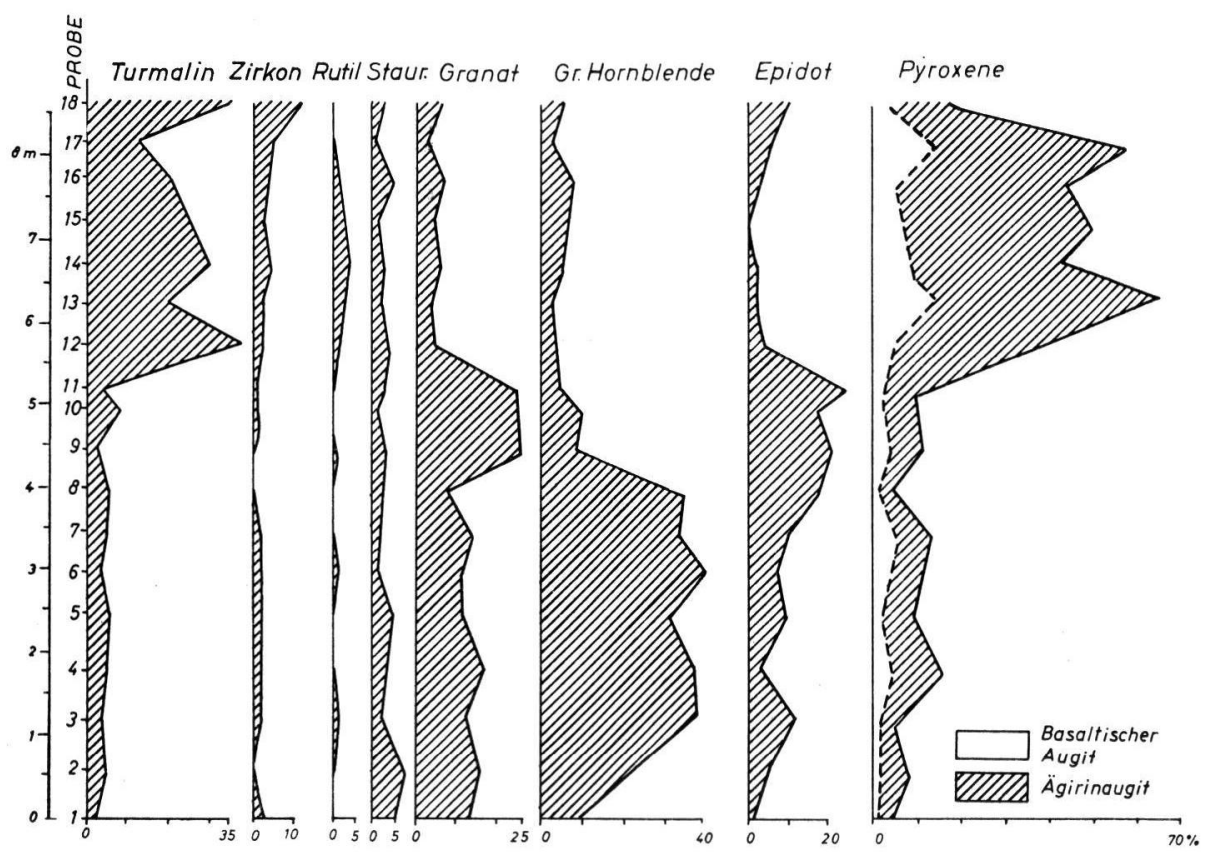

Abb. 6. Verteilung der Schwerminerale in den Schottern der jüngeren Hauptterrasse in der Tongrube Kärlich, Neuwieder Becken. 
lichen Nebenkegel stammen wahrscheinlich die alkalibasaltischen Tuffe, die am Wege nach Rieden im höheren Profil der Selbergittuffe auftreten. Die Tätigkeit des Sulzbusch hat lange angedauert und war im ganzen gleichalterig mit den Ausbrüchen der Selbergittuffe. Der Alterswert wurde an der vom Nebenkegel stammenden Lava ermittelt. Er steht dem Alter der selbergitischen Bimssteintuffe (350.103a) nahe.

Selbergit des Engelner Kopfs und Leucitophyr des Perlerkopfs Alter: $320.10^{3}$ a und $320.10^{3}$ a

Zwischen den Ausbrüchen des Engelner Kopfs und Perlerkopfs und den Rheinterrassen lassen sich keine unmittelbaren Altersbeziehungen herstellen. Die Bestimmungen dienten dem Zweck, die Tätigkeit der selbergitischen Vulkane zeitlich genauer abzugrenzen. Nach der Mineralführung der Rheinterrassen sollten die Ausbrüche der selbergitischen Tuffe und Gesteine im Nordwesten des Laacher-See-Gebietes nicht wesentlich jünger als die jüngere Hauptterrasse sein. Mit den schon behandelten Vorkommen und den Gesteinen vom Engelner Kopf und Perlerkopf ist die Mehrzahl der selbergitischen Fördermassen erfaßt. Die Daten „Engelner Kopf" und „Perlerkopf " schließen sich eng an die vorhergehenden Alter an. Die Ausbrüche der selbergitischen Magmen waren danach kurz nach der Aufschotterung der jüngeren Hauptterrasse im wesentlichen beendet.

Alkalibas alt-Lava des Hochsimmers

Alter: $300.10^{3} a$

Die Lava des Hochsimmers lagert ungefähr $90 \mathrm{~m}$ über dem heutigen Flußbett auf einem alten Talboden der Nette. Die Eintiefung des Nettetales ist durch Einschüttungen von basaltischen und selbergitischen Förderprodukten mehrfach gestört worden und dadurch eine Zeitlang hinter der Entwicklung des Rheintales zurückgeblieben. Die stratigraphische Stellung der Hochsimmer-Lava wurde daher nicht nach ihrer Höhenlage, sondern aufgrund anderer Anhaltspunkte festgelegt.

In den vor der Lavaeffusion ausgeworfenen Tuffen des Hochsimmers finden sich als Auswürflinge selbergitische Bimssteine aus der oberen Bimssteinschicht des Riedener Tuffprofiles. Unter dem Lavastrom treten in Geröllablagerungen der alten Nette Stücke von Sulzbusch-Lava auf. Die Tätigkeit des Hochsimmers begann also nach der Ablagerung der Bimssteintuffe „Hoheley“ und „In der Erle“ und der Effusion der Sulzbusch-Lava, das ist nach $350.10^{3}$ a und $340.10^{3}$ a.

Die Hochsimmertuffe im Liegenden der Lava sind zum Teil in Löß eingeschaltet und wurden somit in einer Kaltzeit gefördert. Hierauf weisen auch bis einige Meter mächtige Lagen von eckigem Solifluktionsschutt unter der Lava hin, der mit Löß vermischt ist. Westlich St. Johann sind drei übereinanderliegende Teilströme des Hochsimmers jeweils durch eine Zwischenlage von Solifluktionsschutt und Löß getrennt. Minerale vom Hochsimmer fehlen noch in der jüngeren Hauptterrasse, treten aber in den Schottern der älteren Mittelterrasse des Rheines bei Hüllenberg auf. Die Ausbrüche des Hochsimmers fanden daher wahrscheinlich am Anfang der Aufschotterung der älteren Mittelterrasse statt.

Alkalibas a l t-Lava des Hochsteins

Alter: $260.10^{3} \mathrm{a}$

Der Hochstein war ebenso wie der Sulzbusch lange tätig. Die von ihm stammenden älteren Lapillituffe unterlagern an der Straße Ettringen-Bell den Selbergittuff der Rodderhöfe. Weitere Tuffe des Hochsteins sind in der Grube „In der Erle“ in die oberen selbergitischen Bimssteintuffe eingeschaltet. Der bis zum Thürer Berg sich erstreckende Lavastrom muß noch bedeutend jünger sein. Zur Zeit der Effusion hatte der Kellbach die Selbergittuffdecke östlich vom Hochstein bereits durchschnitten und auch den devonischen Untergrund schon tiefer erodiert. Die Unterlage der Lava dürfte jedoch bei Obermendig 
und Thür über dem Niveau der mittleren Mittelterrasse liegen. Die petrographische Zusammensetzung des Gesteins leitet schon zu den Basaniten über, die, wie die Laven des Bausenberg, Veitskopf und Lummerfeld, vor allem in der Zeit der mittleren Mittelterrasse gefördert wurden. Das Alter würde den angegebenen Zusammenhängen ungefähr entsprechen.

Lava aus dem Tuff, Leilenkopf III “

Alter: $220.10^{3} \mathrm{a}$

Eine Analyse des Gesteines liegt nicht vor.

In den älteren Tuffen „Leilenkopf I“ und „Leilenkopf II“ ist nach ihrer Ablagerung ein zum Brohtal orientiertes System von Erosionsmulden und -rinnen entstanden, das bis auf das Niveau der älteren Mittelterrasse herabreicht, sich von hier aber nicht weiter nach unten fortsetzt.

In der bei Niederlützingen auslaufenden westlichen Erosionsrinne lagern auf der Erosionsoberfläche Reste eines Lösses mit Lößkindeln, der frühestens in der Kaltzeit der älteren Mittelterrasse abgesetzt worden sein kann. Anschließend wurde er, wahrscheinlich unter Bodenbildung, entkalkt und dann größtenteils wieder abgetragen.

Über dem reliktischen Löß lagern jüngere, lößartige Einwehungen, dann folgen feingeschichtete basaltische Sande, die durch Kryoturbation stark gestört sind. Sie gehen nach oben in sandreichen Lapillituff über, in dem größere Lava-Auswürflinge enthalten sind. Das Profil endigt mit zwei Lößhorizonten, zwischen die würmzeitlicher Wehrer Bimsstein eingeschaltet ist.

Der Sand- und Lapilli-Tuff „Leilenkopf III“ ist nach diesen Zusammenhängen jünger als die ältere Mittelterrasse und älter als die Niederterrasse.

Am Hotel „Kleiner Petersberg“ bei Niederbreisig, $5 \mathrm{~km}$ vom Leilenkopf entfernt, ist eine $60 \mathrm{~cm}$ mächtige Lage des Tuff „Leilenkopf III“ in die Schotter der mittleren Mittelterrasse in $0.9 \mathrm{~m}$ Höhe primär-äolisch eingeschaltet. Die Aufschotterung der mittleren Mittelterrasse hat danach nicht lange vor 220.103a begonnen.

Alkalibasalt-Lava des Ba usenbergs

Alter: $150.10^{3} \mathrm{a}, 140.10^{3} \mathrm{a}$

Bei Gönnersdorf im Vinxttal liegt der Lavastrom des Bausenbergs zum größten Teil einer Verebnung auf, die mit der mittleren Mittelterrasse des Rheintales parallelisiert werden kann. Die Stirn des Stromes befindet sich noch einige Meter unter diesem Niveau. Die Lavaeffusion fand statt, als die mittlere Mittelterrasse schon etwas eingeschnitten war. Das Alter „Bausenberg“ wurde daher in die Zeit zwischen der Bildung der mittleren und unteren Mittelterrasse eingeordnet.

\section{Zuverlässigkeit der K-Ar-Daten}

Wie weit die K-Ar-Daten das wirkliche Alter der Vulkanausbrüche und der jeweils zur selben Zeit in Bildung begriffenen Terrassen wiedergeben, hängt vor allem davon $a b$, ob die Proben bei der Förderung vollständig entgast waren und in welchem Umfange sie das in der Folgezeit sich bildende radiogene Argon behielten. Mit physikalischen Methoden lassen sich diese Fragen in den vorliegenden Fällen nicht beantworten. Die Beurteilung der Zuverlässigkeit der K-Ar-Daten muß sich in der Hauptsache auf die geologischen $\mathrm{Zu}$ sammenhänge stützen, die allerdings nur relative Aussagen gestatten. Diese reichen jedoch häufig aus, um entscheiden zu können, ob ein Alterswert möglich und wahrscheinlich oder mit dem Ablauf der geologischen Ereignisse unvereinbar ist. Mit der zunehmenden Zahl der Bestimmungen ergaben sich auch an Hand der Daten selber Hinweise auf den Grad ihrer Genauigkeit. 
Nach den gegebenen Beurteilungsmöglichkeiten wurden die Daten geschieden in wahrscheinliche und unwahrscheinliche Alter.

Die wahrscheinlichen Alter sind in dem Zeitschema der Terrassen und Eiszeiten in Abb. 8 enthalten. Sie passen sich, wie gezeigt wurde, in den Ablauf sowohl der vulkanischen Vorgänge wie auch der Terrassenbildungen ohne erkennbare Widersprüche ein. Die aus selbergitischen Tuffen und Gesteinen stammenden Proben ergaben die höheren, die Lavaproben die mittleren Alterswerte. Dies entspricht der sedimentpetrographisch festgestellten Mineralverteilung in den Terrassenschottern. Die älteren Vulkanausbrüche ließen sich mit den Hauptterrassen, die danach folgenden mit den Mittelterrassen konnektieren. Die Alterswerte stehen auch mit der engeren Förderfolge im Vulkangebiet in guter Übereinstimmung.

Nach den Beziehungen zur Talmorphologie ist der Vulkanismus in der Westeifel und im Laacher-See-Gebiet im ganzen gleichalterig. Zwei Sanidine aus den ältesten alkalibasaltischen Tuffen am Mühlenberg bei Hohenfels und vom Killer Kopf bei Rockeskyll ergaben die Alter "Hohenfels“ $=460.10^{3} \mathrm{a}$ und „Rockeskyll“ $=430.10^{3} \mathrm{a}$. Sie liegen zwischen den Altern "Schellkopf“ und „Hardt“ (Rieden) des Laacher Gebietes. In der Westeifel haben danach, wie im Laacher-See-Gebiet, schon vor der Aufschotterung der älteren Hauptterrasse Vulkanausbrüche stattgefunden.

Unwahrscheinliche Alter ergaben die Proben:

Alkalibasaltische Laven "Mauerley “ und "Lummerfeld “

Alter: Mauerley $100.10^{3} \mathrm{a}$

Lummerfeld $90.10^{3} \mathrm{a}$

Die Lavaströme „Mauerley“ und „Lummerfeld“ liegen der mittleren Mittelterrasse des Gleeser Tales und Brohltales auf und sollten gegenüber der Lava des Bausenberg das gleiche oder ein etwas höheres Alter besitzen. In den Vorkommen gibt es keine Steinbrüche. Die Proben wurden daher an der Oberfläche entnommen. In der Würmeiszeit sind beide Ströme mehrere Male von Bimssteintuffen eingedeckt worden, die eine Mindesttemperatur zwischen $400-600^{\circ} \mathrm{C}$ besaßen. Möglicherweise ist dadurch nahe der Oberfläche ein Teil des radiogenen Argons aus den Lavagesteinen ausgetrieben worden, so daß die Proben zu geringe Alter ergeben.

Alkalibasaltische Lava des Fornicher Kopf südlich Brohl Alter: $270.10^{3} \mathrm{a}$

Die Lava des Fornicher Kopf ist bis auf die jüngere Mittelterrasse des Rheines geflossen. Für den zu hohen Alterswert läßt sich weder eine vulkanologische noch eine petrographische Begründung geben.

Bei dem Alter „Thürer Wald“ $180.10^{3}$ a kann über die Zuverlässigkeit nichts ausgesagt werden. Die Probe stammt aus einem alkalibasaltischen Lavastrom, der sich im Liegenden der Selbergittuffe am Rande des Riedener Kessels befindet. Die Lava kann nach der Lagerung älter als die Tuffe sein. Es ist aber auch mit der Möglichkeit zu rechnen, daß sie subfusiv unter die Tuffe eingedrungen und dann jünger ist, was dem ermittelten Alter entsprechen könnte.

Sanidine "Leubsdorf “, „Kärlich “ und „La ach “

Alter: Leubsdorf $630.10^{3} a$

Kärlich $2450.10^{3} \mathrm{a}$

Laach $230.10^{3} \mathrm{a}$

Laach $\quad 1510.10^{3} \mathrm{a}$

Die Sanidine „Leubsdorf“ stammen aus einem phonolitischen Bimssteintuff, der primärvulkanisch in die Schotter der mittleren Mittelterrasse eingeschaltet ist, die Sanidine „Kär- 
lich" aus würmzeitlichem Wehrer Bimsstein in der Tongrube Kärlich und die Sanidine „Laach“ aus dem allerödzeitlichen Bimsstein des Laacher Kessels. Die Daten sind mit der relativen Abfolge der Tuffe nicht vereinbar und im ganzen zu hoch.

Die Sanidine „Leubsdorf“ und „Kärlich“ treten als lose, einige mm große Kristalle in den Ablagerungen auf. Sie sind teils endomagmatisch gebildet worden, zum Teil stammen sie wahrscheinlich aus Sanidiniten, die kontaktmetasomatisch aus vordevonischen Phylliten und Glimmerschiefern im Untergrund des Rheinischen Schiefergebirges hervorgegangen sind. Die zu hohen Alter dürften auf ererbtes Argon in den aus Sanidiniten stammenden Kristallen zurückzuführen sein. Das gleiche kann bei den Sanidinen „Laach“ angenommen werden, die aus metasomatisch entstandenen Sanidiniten separiert worden sind. Die Temperatur der Bimssteintuffe betrug bei der Eruption mindestens einige $100^{\circ} \mathrm{C}$. Die Ausbrüche erfolgten sehr rasch. Wahrscheinlich wurde hierbei nicht alles radiogene Argon ausgetrieben.

Größere Einsprenglingskristalle von Biotit und Augit aus Tuffen und Gesteinen

$\begin{array}{lr}\text { Alter: Biotit "Leilenkopf II" } & 1300.10^{3} \mathrm{a} \\ \text { Biotit "Hoheley“ } & 2500.10^{3} \mathrm{a} \\ \text { Biotit "Thürer Wald" } & 1500.10^{3} \mathrm{a} \\ \text { Biotit "Leilenkopf III" } & 1500.10^{3} \mathrm{a} \\ \text { Biotit "Fornicher Kopf " } & 280.10^{3} \mathrm{a} \\ \text { Augit "Bausenberg" } & 11000.10^{3} \mathrm{a} \\ \text { Augit "Fornicher Kopf " } & 3500.10^{3} \mathrm{a}\end{array}$

Die Einsprenglinge von Biotit und Augit ergaben gegenüber den aus denselben Vorkommen stammenden Sanidinen und Gesteinen stets höhere und in der Mehrzahl stärker abweichende Alter. Es handelt sich bei ihnen um intratellurische Frühausscheidungen der vulkanischen Magmen, die mehr oder weniger lange vor den Vulkanausbrüchen kristallisierten und ein höheres Alter als diese besitzen. Die Altersmessungen an den Einsprenglingen können dann zwar richtig sein, die bestimmten Werte dürfen aber nicht mit dem Alter der Vulkanausbrüche gleichgesetzt werden. Ob die Altersunterschiede zwischen den Biotitund Augiteinsprenglingen und ihren erst nach den Ausbrüchen erstarrten Wirtgesteinen hierauf zurückzuführen ist, läßt sich noch nicht entscheiden. Jedenfalls dürften nach den vorliegenden Ergebnissen frühgebildete Einsprenglinge von Biotit, Augit und wahrscheinlich auch von Hornblende für die Datierung junger Vulkanausbrüche und von Sedimenten, die mit den Ausbrüchen zeitlich konnektiert werden können, wie im vorliegenden Falle die Schotter der Rheinterrassen, nicht geeignet sein.

\section{K-Ar-Daten und Eiszeitchronologie}

Das System der pleistozänen Flußterrassen bildet eine natürliche und, solange die Unterscheidung nur nach der Höhenlage durchgeführt wird, hypothesenfreie Grundlage für die stratigraphische Festlegung der K-Ar-Daten. Versucht man nun unter der Annahme, daß die Schotterdecken der einzelnen Terrassen jeweils einer besonderen Eiszeit entsprechen, die Daten mit dem Ablauf der pleistozänen Vereisungen in Verbindung zu bringen, so ergibt sich, daß das Terrassenschema mit der herkömmlichen Einteilung der Glacialzeiten nach PENCK und BRÜCKNER nicht vollständig in Einklang gebracht werden kann. Dies führt zu der Frage, wie weit die einzelnen Terrassen und Schotterhorizonte Glacialzeiten oder möglicherweise auch zeitlich weiter auseinanderliegenden Stadialen einer Glacialzeit angehören.

An der Verteilung von Olivin, Augit und Sanidin, die aus Tuffen stammen, die gleichzeitig in einzelnen Horizonten des Würm-Löß-Profiles in der Tongrube Kärlich und in den Schottern der Niederterrasse im Neuwieder Becken vulkanisch-äolisch abgelagert wor- 
den sind, ließ sich der Nachweis erbringen, daß die Schotterdecke der Niederterrasse in ihren verschiedenen Höhen mit einzelnen Horizonten des Würm-Löß-Profiles korreliert werden kann (Abb. 7). Die Gesamtschotterdecke besteht danach aus mehreren durch Sandschichten getrennten Teildecken, die in den aufeinanderfolgenden Stadialen der Würmeiszeit abgelagert wurden. Daraus folgt, daß das vollständige Schotterprofil der Niederterrasse dem Gesamtzeitraum der Würmeiszeit ungefähr gleichgestellt werden kann. Entsprechendes gilt auch für die übrigen Terrassenschotter. So lagern in der Schotterdecke der mittleren Mittelterrasse mindestens zwei Teildecken übereinander, zwischen die, wie in der Niederterrasse, eine zum Teil lößartige Sandschicht zwischengeschaltet ist. Aufgrund dieser Befunde kann man annehmen, daß zwischen den Stadialen einer Eiszeit keine so weitgehende Taleintiefung stattgefunden hat, daß sie den jetzigen Abständen zwischen den Rheinterrassen gleich käme. Die Schotterdecken der einzelnen Terrassen werden daher jeweils einer selbständigen Eiszeit zugeordnet.

Es wird jetzt als sicher angenommen, daß die Niederterrassen der Würmeiszeit und die untere Mittelterrasse der Rißeiszeit entspricht (WoLDSTEDT 1961). Die Hauptterrasse wird im allgemeinen mit der Günzeiszeit in Zusammenhang gebracht. Wenn den einzelnen, durch längere Erosionszeiten getrennten Terrassenbildungen jeweils eine Eiszeit entspricht, sind zwischen Günz und Riß außer der Mindeleiszeit noch zwei weitere Eiszeiten einzu-

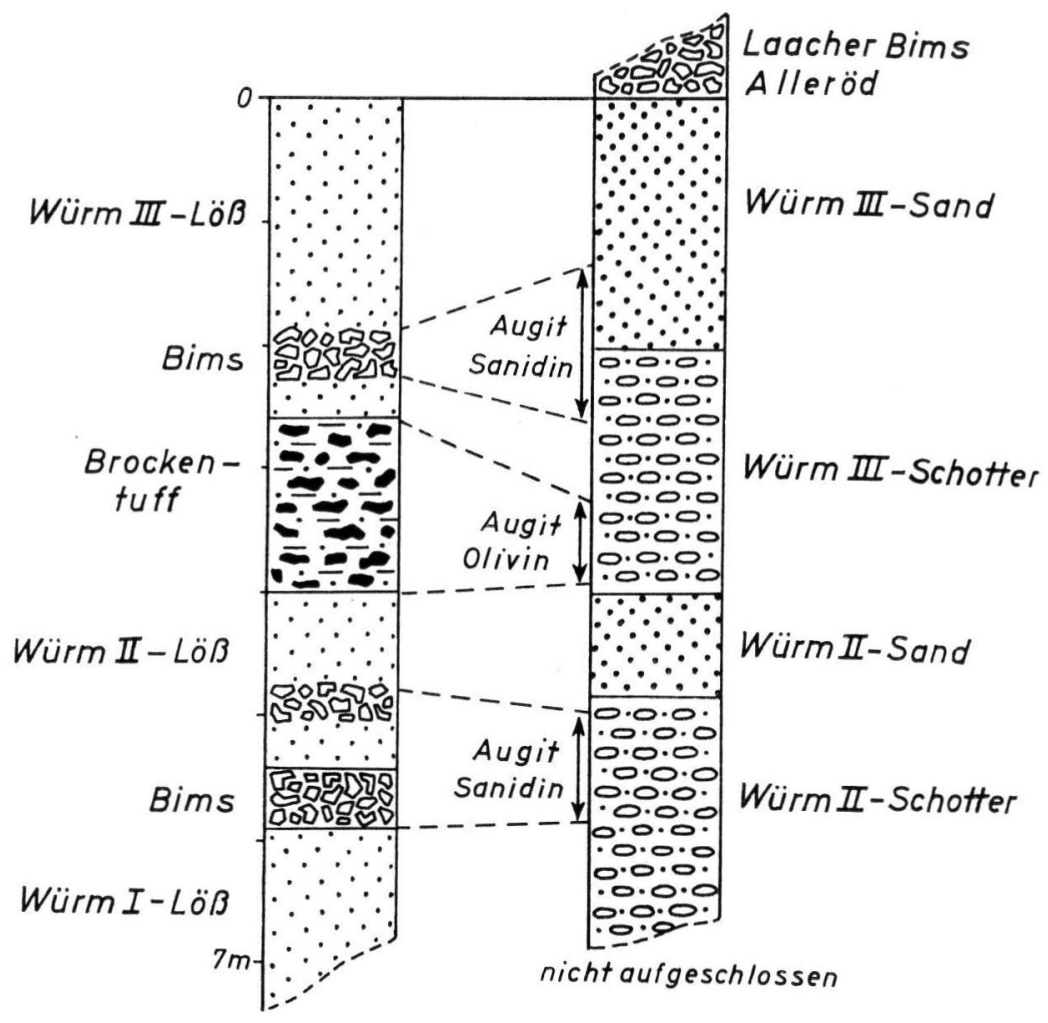

\section{Lößprofil Kärlich NT-Profil Urmitz (nach ROSAUER 1957)}

Abb. 7. Zusammenhang zwischen den vulkanischen Tuffeinschaltungen im Würmlöß der Tongrube Kärlich und dem Mineralgehalt der Schotter der Niederterrasse des Rheines bei Urmitz im Neuwieder Becken. 


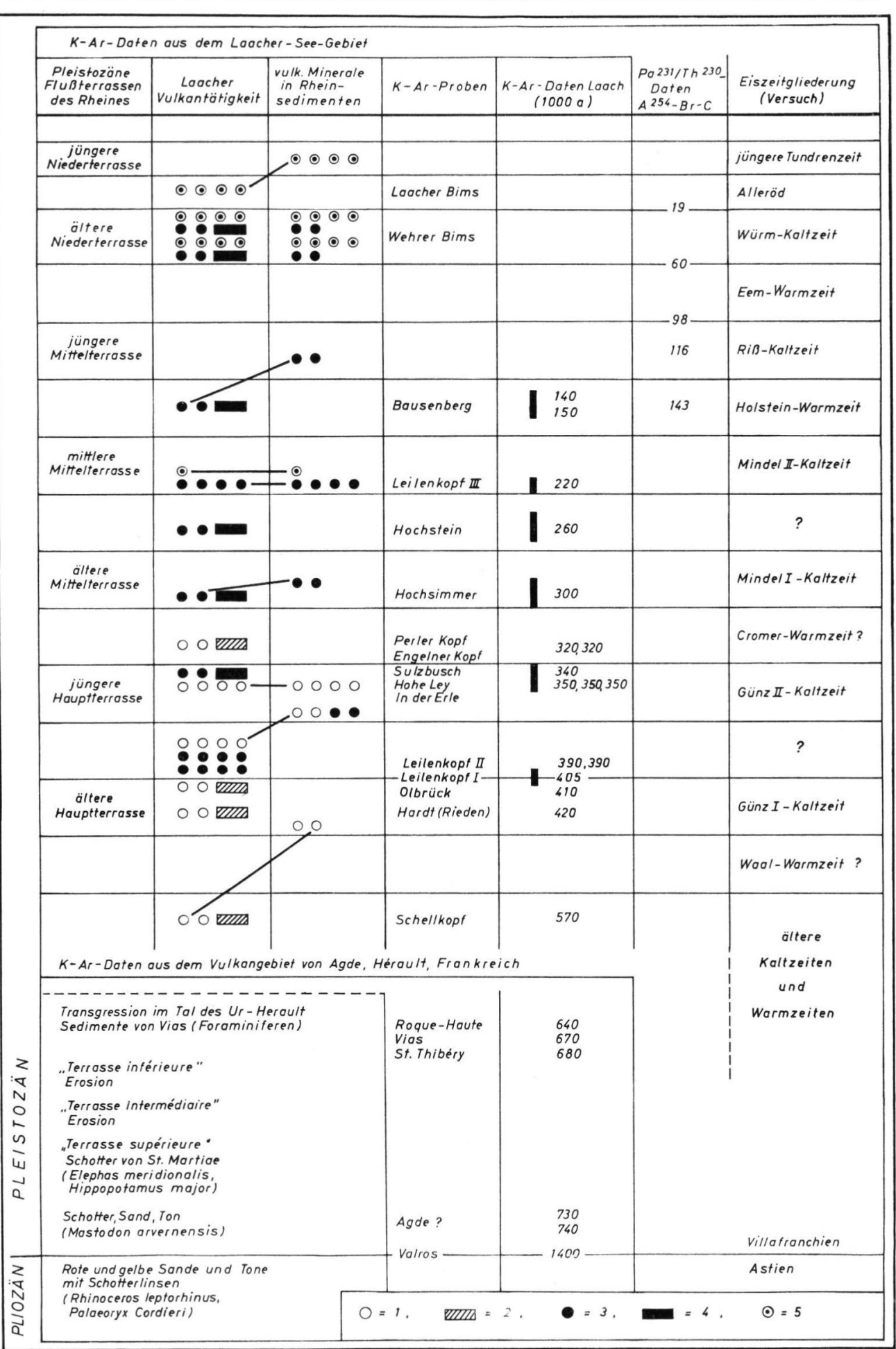

Abb. 8. Zeittafel des Laacher Vulkanismus, der Schotterterrassen des Rheines und der Eiszeiten, sowie Verbindungen der vulkanischen Förderprodukte zu den Schotterablagerungen des Rheines. 1: Selbergittuff; 2: Selbergit; 3: Basalttuff; 4: Basaltlava; 5: Bimsstein. 
schalten, die bis jetzt noch unbekannt sind. In dem hier aufgestellten Zeitschema der Eiszeiten (Abb. 8) sind die Günz- und Mindeleiszeit vorläufig in die Kaltzeiten Günz I und II und Mindel I und II aufgeteilt worden.

In Abhängigkeit von den Gegebenheiten im Gelände konnte die stratigraphische Zuordnung der K-Ar-Daten zu den Terrassen und damit zum Ablauf der Eiszeiten mit größerer oder geringerer Genauigkeit erfolgen. Dementsprechend sind die Bereiche, in welche die Alterswerte wahrscheinlich zu stellen sind, in unterschiedlicher Länge dargestellt.

Die in das Zeitschema eingetragenen $\mathrm{Pa}^{231} / \mathrm{Th}^{230}$-Daten von Rosholt, EMILIANI, Geiss, Koczy \& WANGERsKy (1962) treffen sich im Bereich der Holsteinwarmzeit mit den K-ArDaten aus dem Laacher Vulkangebiet.

Nach den $\mathrm{Pa}^{231} / \mathrm{Th}^{230}$-Bestimmungen betrug die mittlere Dauer der Glacialzeiten Würm und Riß und des Eem-Interglacials jeweils ungefähr 40.103a. Eine ähnliche Größenordnung ergibt sich für die vorausgehenden Glacial- und Interglacialzeiten aus den Laacher Daten. Vom Alter „Leilenkopf III“ am Anfang der Mindel II-Kaltzeit und „Hochsimmer" am Anfang der Mindel I-Kaltzeit beträgt das Mittel bis zum Ende des Würms jeweils rund 40.103a, vom Alter „Olbrück“ - „Leilenkopf I“ am Übergang vom Günz I zum Interglacial Günz I - Günz II bis zum Ende des Würms etwa 39.103a und zwischen „Olbrück“ - „Leilenkopf I“ und „Leilenkopf III“ 37-38.103a. Von der Günz I-Kaltzeit an dürfte danach die mittlere Dauer der Glacial- und Interglacialzeiten nahe bei $40.10^{3}$ a gelegen haben.

In das Zeitschema wurden auch die an Lava-Proben aus dem Vulkangebiet von Agde, Dept. Hérault, Südfrankreich ermittelten Alter zusammen mit den stratigraphischen Angaben von J. B. KLoostermann (1959) aufgenommen. Aus der Schichtausbildung ergeben sich Hinweise auf eine Abfolge von Kalt- und Warmzeiten, die aufgrund der Alterswerte älter als die Günz I-Kaltzeit sind. Evernden \& al. (1957) konnten auch in Californien eine Kaltzeit bestimmen, die älter als $960.10^{3} \mathrm{a}$ ist. Die Grenze Pliozän/Pleistozän liegt nach dem Alter "Valros“ bei 1400.103a, nach einer von Evernden (1964) am selben Gestein vorgenommenen Bestimmung bei $1600.10^{3} \mathrm{a}$.

A nerkennungen : Die Autoren danken dem Direktor des MPI für Kernphysik in Heidelberg, Herrn Prof. Dr. Gentner, für sein förderndes Interesse an der Durchführung dieser Arbeit und die Bereitstellung der notwendigen Institutsgeräte. Weiterer Dank gilt Herrn Prof. Dr. Woldstedt, Bonn, für fördernde Diskussionen. H. J. Lippolt dankt Herrn H. Richter für seine Hilfe bei der Durchführung der K-Analysen und dem Reaktor München für die Bestrahlungen der Biotitproben.

\section{Literatur}

Ahrens, W.: Geologisches Wanderbuch durch das Vulkangebiet des Laacher Sees in der Eifel, Stuttgart 1930.

Amirkhanov, K. I., Brandt, S. B. and BartnitskiI: Diffusion of radiogenic argon in feldspars Doklady Akd. Nauk, SSSR 125, 1345. 1958.

Baadsgaard, H., Lipson, J. and Folinsbee, R. E.: The leakage of radiogenic argon from sanidine Geochim. et Cosmochim. Acta 25, 147. 1961.

Curtis, G. H., Lipson, J. and Evernden, J. L.: Potassium-Argon-dating of Plio-Pleistocene intrusiv rocks. - Nature 178, 1380. 1956.

Dalrymple, G. B.: Potassium argon dates of some Cenozoic volcanic rocks of the Sierra Nevada, California. - Bull. Geol. Soc. Amer., 74, 379. 1963.

Evernden, J. F., Curtis, G. H. and Lipson, J.: Potassium-argon-dating of igneous rocks. Bull. Am. Ass. Petrol. Geol. 41, 2120-2127. 1957.

Evernden, J. F., Curtis, G. H., Kistler, R. W. and Овradovich, J.: Argon Diffusion in glauconite, microcline, sanidine, leucite and phlogopite. - Am. J. Sci. 258, 583. 1960.

Evernden, J. F., Curtis, G. H., Kistler, R.: Potassium Argon Dating of pleistocene Volcanics. Quaternaria 4, 1-5, 1957. 
Fechtig, H., Gentner, W. und Zähringer, J.: Diffusionsverluste von Argon in Mineralien und ihre Auswirkung auf die Kalium-Argon-Altersbestimmung. - Geochim. et Cosmochim. Acta, 19, 70-79. 1960.

FrECHEN, J.: Führer zu vulkanologisch-petrographischen Exkursionen im Siebengebirge am Rhein, Laacher Vulkangebiet und Maargebiet der Westeifel, Stuttgart. 1962.

Frechen, J. und v. D. Boom, G.: Die sedimentpetrographische Horizontierung der pleistozänen Terrassenschotter im Mittelrheingebiet. - Fortschr. Geol. Rheinland u. Westf., 4, 89-125. 1959.

Gentner, W. und Zähringer, J.: Das Kalium-Argon-Alter von Tektiten. - Z. Naturforschung, 15 a, 93. 1960.

Hart, S. R. and Dodd, R. T.: Excess radiogenic Argon in Pyroxenes. - J. Geophys. Research 67, 2998. 1962.

Kloostermann, J. B.: Le Volcanisme de la region d'Agde, Hérault, France. - Diss. Utrecht. 1957.

von Koenigswald, G. H. R., Gentner, W. und Lippolt, H. J.: Age of the Basalt flow at Olduvai, East Africa. - Nature 192. 720. 1961.

Lippolt, H. J.: Kalium-Argon-Datierungen bei kleinen Kalium- und Argon-Konzentrationen. Diss. Heidelberg. 1961.

Lippolt, H. J., Gentner, W. und Wimmenauer, W.: Altersbestimmungen nach der Kalium-ArgonMethode an tertiären Eruptivgesteinen Südwestdeutschlands. - Jh. geol. LA Baden-Württemberg, 6, 507-538. 1964.

Leakey, L. S. B., Evernden, J. F., Curtis, G. H.: Age of Bed I Olduvai Gorge, Tanganyika. Nature 191, 478. 1961.

REYNOLDS, J. H.: Comparative study of argon content and argon diffusion in mica and feldspar. Geochim. et Cosmochim. Acta 12, 177-184. 1957.

Rosholt, J. N., Emiliani, C., Geiss, J., Koczy, F. F., WANGersky, P. J.: P231/Th230 Dating and 018/016 temperature analysis of core A 254-Br-C. - J. Geophys. Research, 67, 2907-2911. 1962.

Woldstedt, P.: Das Eiszeitalter, 1. Bd., Stuttgart, 1961, 2. Bd., Stuttgart, 1958.

ZäHringer, J.: K-Ar-Measurements of tektites, in „Radioactive Dating“, IAEA Wien. 1963.

Manuskr. eingeg. 30. 3. 1965.

Anschrift der Verf.: Prof. Dr. Frechen, Bonn, Poppelsdorfer Schloß, Mineral.-Petrolog. Institut der Universität; Dr. H. J. Lippolt, Heidelberg, Max-Planck-Institut für Kernphysik. 